\title{
The amino acid transporter CG1139 is required for retrograde transport and fast recovery of gut enterocytes after Serratia marcescens intestinal infection
}

\section{Authors}

Catherine Socha ${ }^{1 \S \$}$, Inês S. Pais ${ }^{1 \S}$, Kwang-Zin Lee ${ }^{1 £}$, Matthieu Lestradet ${ }^{1}$, Dominique Ferrandon ${ }^{1,2^{*}}$

1 - Université de Strasbourg, CNRS, RIDI UPR 9022, 67000 Strasbourg, France

2 - Sino-French Hoffmann Institute, Guangzhou Medical University, Guangzhou, China

$\S$ Both authors contributed equally to this work

\$: Present address: 119 Paget Road, CB2 9JG Cambridge UK

$£$ : Present address: Fraunhofer Institute for Molecular Biology and Applied Ecology, Giessen, Germany

*Correspondence: D.Ferrandon@ibmc-cnrs.unistra.fr 


\section{ABSTRACT}

The intestinal tract is constantly exposed to microbes. Severe infections can arise following the ingestion of pathogenic microbes from contaminating food or water sources. The host directly fights off ingested pathogens with resistance mechanisms, the immune response, or withstands and repairs the damages inflicted either by virulence factors or the host immune effectors, through tolerance/resilience mechanisms. In a previous study, we reported the existence in Drosophila melanogaster of a novel evolutionarily conserved resilience mechanism to intestinal infections with a hemolysin-positive Serratia marcescens strain $(S m \mathrm{Db} 11)$, the purge of the apical cytoplasm of enterocytes. The epithelium becomes very thin and recovers rapidly, regaining its normal thickness within several hours. Here, we found that this recovery of gut enterocyte morphology is based on the host internal reserves and not on ingested food. Indeed, we observed a retrograde transport of amino acids from the host hemolymph to the enterocytes. We have identified several amino acid transporters required for recovery and we focused on the SLC36 family transporter CG1139. CG1139 is required for the retrograde transport of amino acids. RNA sequencing revealed that genes involved in the positive regulation of growth were observed in wild-type but not CG1139 mutant guts, in which the expression of $M y c$ and genes involved in Insulin signaling is down-regulated. Functional analysis revealed that $M y c$ is also required for the recovery of the thick gut epithelium after infection. Altogether, our results show the importance of an amino acid transporter in the fast regrowth of the enterocytes upon infection. Unexpectedly, we found that this transporter acts non cell-autonomously and can regulate the transcription of other genes, suggesting a signaling function of CG1139 that therefore appears to act as a transceptor. 


\section{Introduction}

The intestinal tract is one of the largest interfaces between the host body and the external environment, harboring a diverse microbial community that is mostly beneficial to the host (Flint, Scott, Louis, \& Duncan, 2012). However, the proliferation of existing opportunistic pathogens as well as the exposure to food-borne pathogens can severely compromise host health (Ryu et al., 2008). Resistance and resilience mechanisms in the host have been selected during evolution that ensure the homeostasis of the epithelium and limit damages inflicted by pathogens (Ferrandon, 2013). Whereas resistance mechanisms are responsible for decreasing the pathogen burden, resilience mechanisms are responsible for enduring the presence of pathogens. Drosophila melanogaster is a powerful model organism to study such mechanisms, especially due to the power of its sophisticated genetic tools. The regulation of intestinal stem cells (ISCs) is an integrated response required for gut homeostasis and for the replacement of the gut enterocytes that are lost over time (Bonfini, Liu, \& Buchon, 2016).

Amino acids have also been shown to be important in the outcome of an infection (Zhu, Deng, \& Yin, 2018). Amino acid metabolism affects the host physiology and may serve as an energy source for the cell. In mammals, 59 amino acid transporters have been identified and categorized in 12 different Solute Carrier (SLC) families (Thwaites \& Anderson, 2011) such as the Proton-coupled Amino acid Transporters (SLC36 or PATs), the Sodium-coupled Neutral Amino acid Transporters (SLC38 or SNATs), the cationic (SLC7 or CATs) and the heterodimeric (SLC7/SCL3 or HATs) amino acid transporters. In Drosophila, among the average 14000 genes, 603 are predicted to encode putative transporter proteins, which correspond to $4 \%$ of the genome (Featherstone, 2011). Amino acid transporters have redundant functions, as different transporters can carry the same amino acid. These characteristics allow adaptation to various environmental conditions. 
Serratia marcescens $(\mathrm{Sm})$ is a Gram-negative opportunistic human pathogen that secretes several virulence factors, such as proteases and pore-forming toxins. Hemocyte-mediated phagocytosis in the body cavity is an effective response against the ingested $\mathrm{Sm}$ bacteria that manage to cross the intestinal barrier. When injected directly in the hemolymph, $S m$ can resist the systemic immune response essentially because of the O-antigen of its LPS (C. L. Kurz et al., 2003; Nehme et al., 2007). Several genes required for susceptibility or for resistance against ingested $\mathrm{Sm}$ have been identified before in a genome-wide screen. The JAK-STAT pathway was shown to be required for host defence by regulating the proliferation of ISCs (Cronin et al., 2009), a result independently obtained by other investigators (Apidianakis, Pitsouli, Perrimon, \& Rahme, 2009; N Buchon, Broderick, Chakrabarti, \& Lemaitre, 2009; Jiang et al., 2009).

We have previously reported the cytoplasmic purge of enterocytes, a very fast response of the gut epithelium to hemolysin expressing Serratia marcescens (SmDb11) (Lee et al., 2016). After ingestion, the enterocytes of the midgut extrude a considerable portion of their cytoplasm through an aperture that is formed in the apical part of the cell. This happens at very early stages, around one hour upon infection. Consequently, the cells become very thin and flat; enterocytes of the R2 region lose their dome-shaped apical domains typical of this portion of the midgut. Strikingly, the cytoplasmic purge prevents damages to enterocytes as there is no increased enterocyte cell death nor compensatory stem cell proliferation. Moreover, the same cells are able to recover their normal shape and size within the first 20 hours post infection. Damaged organelles such as mitochondria and also likely toxins and invading bacteria are actively extruded out of the exposed enterocytes. We have previously identified $C y c l i n J(C y c J)$ as being required for the recovery of the cell size and shape (Lee et al., 2016). However, it remains unclear how the host can recover from a very thin epithelium that lost a massive part of its cytoplasm and associated organelles and reconstitute the normal 
cell shape and size. Here, we explore this question and show that a retrograde transport of metabolites occurs from the hemolymph to the enterocytes during the recovery of the epithelium. We found that the amino acid transporter CG1139 localizes basally in the labyrinth region of the enterocytes, a zone of intensive exchange between the enterocyte and the hemolymph, and is required for the retrograde transport of a modified amino-acid. Moreover, CG1139 is required for the fast regrowth of the epithelium, but a recovery is still possible in its absence, albeit slower, suggesting a possible redundancy with other amino acid transporters also shown to be involved in the regrowth of thinned enterocytes. Unexpectedly for a transmembrane protein, we found CG1139 to act non-cell autonomously and we suggest that it may be involved in cell to cell signaling and possibly inter-organ communication.

\section{Results}

\section{A retrograde transport from the hemolymph to the gut takes place upon infection}

When flies are orally infected with $S$. marcescens that express hemolysin ( $S m \mathrm{Db} 11)$, midgut enterocytes expel their apical cytoplasm, which can be observed as large cytoplasmic extrusions forming in the ectoperitrophic space. The cells subsequently experience a considerable decrease in their thickness and volume, as well as a lowered microvilli density and size (Lee et al., 2016). As a consequence of this cytoplasmic purge, these cells lose a fraction of their components and organelles, such as lipids and mitochondria (Lee et al., 2016). Strikingly, the recovery to the normal shape and volume occurs relatively rapidly, being completely restored around $16 \mathrm{~h}$ to $24 \mathrm{~h}$ after infection. We started by investigating the origin of the reserves that are required for the intestinal epithelium recovery. We usually infect the flies with $\mathrm{SmDb} 11$ in a $50 \mathrm{mM}$ sucrose solution containing $10 \%$ of Lysogeny broth medium (LB). LB is a nutritive medium containing peptides and vitamins that sustains bacterial growth. To analyze a possible role of LB in the recovery and growth of the gut 
enterocytes after infection in an anterograde manner, a.k.a, direct absorption of nutrients by the intestinal epithelium, we replaced LB by PBS and analyzed if the flies recovered to the same extent as the control infected flies fed sucrose solution with $10 \%$ LB. Three hours postinfection the cytoplasmic purge occurred to the same extent in both conditions leading to a very thin intestinal epithelium of the gut (Figure 1A). At 16h post-infection, the gut enterocytes were able to recover equally well under both conditions (Figure 1A). We also replaced LB by a mix of essential and non-essential amino acids in the infection solution as a further positive control and obtained similar results (Figure 1A). These results suggest that the presence of LB in the infection solution is not required for the recovery of the gut enterocytes. Because flies were still feeding on sucrose during infection, we decided to analyze if sucrose itself could have some role in the recovery. For this purpose, we exposed the flies to $S m \mathrm{Db} 11$ re-suspended in a PBS1x solution for $3 \mathrm{~h}$ in the absence of any sucrose or LB; after that period of exposure to bacteria, we flipped the flies to sterile PBS1x, to H2O or to sucrose with LB as a control for $13 \mathrm{~h}$. Surprisingly, almost all the flies in both conditions were able to restore a thick epithelium in the absence of any external nutrients (Figure 1B, 1C, Supplementary Figure 1A). These results suggest that upon infection, the fast recovery of the gut epithelial cells from the cytoplasmic purge is not dependent on newly acquired nutrients, but rather on metabolites stored in the insect body.

To explore this possibility, we decided to starve flies for $24 \mathrm{~h}$ in $\mathrm{H} 2 \mathrm{O}$ to deplete their internal metabolic stores and then to observe how well they would recover from the cytoplasmic purge. Only $10 \%$ of the flies were able to recover to a normal epithelium, while the others $80 \%$ and $10 \%$ displayed respectively a semi-thin and thin epithelium (Figure 1D, E). These results show that when flies are pre-starved before the infection, the gut epithelium cannot recover to the same extent as nonstarved flies $16 \mathrm{~h}$ post-infection. 
We reasoned that after infection a retrograde transport of metabolites would be occurring from the rest of the body to the gut to sustain the fast recovery of the epithelium to its normal thickness. We first measured the level of free amino acids in the hemolymph after bacterial infection. At $3 \mathrm{~h}$ post-infection, we observed an increase of free amino acids in the hemolymph of flies. This increase was transient since it was no longer detected $6 \mathrm{~h}$ postinfection (Figure 1F). This observation led us to ask whether amino acids in the hemolymph might be transported to the gut in a retrograde manner during the recovery process. To answer this question, we used the Click-iT AHA (L-azidohomoalanine) technique, which employs a modified amino acid that is an analog of methionine recognized by the translation machinery and incorporated into newly synthetized proteins. After reacting AHA with an alkyne fluorophore probe, the incorporated analog can be detected by fluorescence in tissues in which translation is taking place (Supplementary Figure 1B). We injected AHA in the hemocoel of flies. Next, we exposed the injected flies to either standard fly food, $S m \mathrm{Db} 11$, sucrose or to water (starved flies) for $6 \mathrm{~h}$. Flies placed on food presented very low or absent fluorescence in the gut in contrast to infected flies, which presented a large increase in the midgut epithelium fluorescence 6h post-infection (Figure 1G). Interestingly, flies that were placed in sucrose or starved in water also displayed an increase of the midgut epithelium fluorescence (Figure 1G). These results show that the modified injected amino acid is being taken up from the hemolymph and incorporated into the enterocytes during the recovery phase of cytoplasmic extrusion or upon amino-acid starvation. Taken together, these experiments suggest the occurrence of a retrograde transport to the midgut epithelium in response to infection or starvation.

The amino acid transporter CG1139 is required for the fast recovery from the gut epithelium after the cytoplasmic purge 
Because there is an increase in free amino acids in the hemolymph and a retrograde transport to the gut enterocytes upon infection, we next asked if gut amino acid transporters are involved in epithelium recovery upon infection. We screened 28 putative amino acids transporters expressed in the Drosophila midgut by knocking down their gene expression in enterocytes using the Gal4/UAS system with a specific driver (NP1-Gal4, Gal $\left.80^{\text {ts }}=\mathrm{NP}\right)$ and analyzed how the corresponding midguts recover from infection. Interestingly, the knockdown of several amino acid transporter genes in the gut resulted in an impaired recovery of the gut epithelium after the cytoplasmic purge (Supplementary Figure 2A), suggesting their involvement in this process. Remarkably, one of the amino acid transporters hit that presented the strongest phenotype with more than $50 \%$ of the guts with an epithelium remaining thin, CG1139, was the sole transporter gene found to be up-regulated $6 \mathrm{~h}$ postinfection in an RNA sequencing experiment that was previously performed in our laboratory (Figure 2A). We validated the RNA sequencing results by RT-qPCR and we observed a more than 10-fold up-regulation of CG1139 transcripts at 9h post-infection (Figure 2B). We also analyzed the expression of CG1139 upon infection with $S m 21 C 4$, a S. marcescens mutant for hemolysin that does not cause an accentuated cytoplasmic purge (Lee et al., 2016). Although CG1139 was still somewhat induced, it was not as strongly expressed as after SmDb11 challenge (Figure 2B).

CG1139 encodes an amino acid transporter similar to a mammalian PAT (Proton-assisted SLC36 Amino acid Transporter) (Supplementary Figure 2B) and it has previously been shown in D. melanogaster to be involved in cell growth (Goberdhan, Meredith, Boyd, \& Wilson, 2005). The possibility that this transporter might regulate the recovery phase that follows the cytoplasmic purge prompted us to investigate further the role of this specific transporter in enterocytes. 
Having validated the efficiency of $N P>C G 1139$ RNAi (Supplementary Figure 3A), we next confirmed the results from the mini-screen of amino acid transporters by analyzing the gut epithelium recovery of $N P>C G 1139$ RNAi flies $16 \mathrm{~h}$ and $48 \mathrm{~h}$ post-infection. Both $N P>C G 1139$ RNAi and control flies showed a cytoplasmic purge at $3 \mathrm{~h}$, with thin epithelium of the gut enterocytes. After $16 \mathrm{~h}$, we confirmed that almost all the control flies had recovered a normal gut epithelium thickness. In contrast, only $10 \%$ of $N P>C G 1139$ RNAi flies had restored the enterocytes back to their normal size, with 50\% of the flies still presenting a very thin epithelium of the gut (Figure 2C, D). However, 48h post-infection NP>CG1139 RNAi flies had recovered completely, suggesting that the absence of this transporter causes a delay in the recovery. We obtained similar results using an independent RNAi line, and we also performed a rescue experiment with the overexpression of a transgene encoding a CG1139$G F P$ fusion in the NP>CG1139 RNAi background. The expression of a wild-type copy of CG1139 was sufficient to reinstate the recovery by $16 \mathrm{~h}$ even though a fusion protein was used (Supplementary Figure 3B, Figure 2E). To limit possible effects of the genetic background of CG1139 RNAi, we isogenized this line into the $w^{1118}$ iso background and measured more precisely the enterocytes thickness. Overall, we obtained similar results to those obtained with the non-isogenized lines reported so far. In sucrose, both NP>iso and NP>CG1139 RNAi iso presented enterocytes with a thickness around $22 \mu \mathrm{m}$. At $3 \mathrm{~h}$ post-infection, when the cytoplasmic purge had occurred, the enterocytes had remarkably lost some $15 \mu \mathrm{m}$ thickness (median of $7 \mu \mathrm{m}$ ) (Figure 2F). At 16h post-infection, while control flies had recovered their size (median of $25.5 \mu \mathrm{m}$ ), enterocytes from NP>CG1139 RNAi iso remained thin (median of $10.5 \mu \mathrm{m})$, not different from $\operatorname{SmDb} 11$ at $3 \mathrm{~h}(\operatorname{lm}, p=0.1023)$.

In addition to RNAi, we used a CRISPR mutant for CG1139 to confirm the delayed recovery of the gut observed with RNAi lines (Supplementary Figure 3C). After infection, there is no up-regulation of CG1139 in the mutant, similar to $N P>C G 1139$ RNAi (Supplementary 
Figure 3D). We confirmed that the mutant also presented a delay in the recovery after infection, with the gut enterocytes remaining thin at $16 \mathrm{~h}$ but recovering after $48 \mathrm{~h}$, similar to CG1139 knockdown in the enterocytes (Figure 2G). All together, these results show that CG1139 is an amino acid transporter induced in the gut upon infection and required in the enterocytes for the fast recovery of the gut epithelium after the cytoplasmic purge.

High-Throughput expression data from Flybase shows that CG1139 is expressed in the adult midgut, but it is also very highly expressed in the Malpighian tubules. In addition, data from Flygut-seq shows that CG1139 is also expressed in the visceral muscles surrounding the gut. Therefore, we decided to knockdown CG1139 in tissues and cell types other than enterocytes to observe if the role in recovery would be restricted to this intestinal epithelium cell type. We

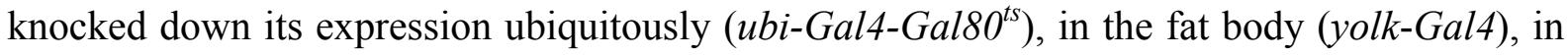
the Malpighian tubules (uro-Gal4), in the visceral muscles (how-Gal4), in the stem cells (esgGal4) and in the enteroendocrine cells (pros-Gal4). When CG1139 was knocked down ubiquitously ( $u b i>C G 1139$ RNAi), the flies presented a similarly thin epithelium at $16 \mathrm{~h}$ postinfection as $N P>C G 1139$ RNAi flies (Supplementary Figure 4A). None of the other flies displayed an impaired recovery when CG1139 was knocked down in the corresponding tissues (Supplementary Figure 4A-C), except for the esg-Gal4 where CG1139 was downregulated in epithelial progenitor cells (Figure 2H, Supplementary Figure 4D). More than $70 \%$ of esg-Gal4>CG1139 RNAi flies displayed a thin epithelium $16 \mathrm{~h}$ post infection (Figure 2H). These results suggest that either CG1139 is also required in the progenitor cells of the gut epithelium during recovery or that during differentiation the new enterocytes generated during the RNAi induction period that lasts between 5 to 7 days lack the transporter as well.

To visualize the tissues and the cell types in which CG1139 may be expressed, we used as reporter a $U A S-G F P$ transgene crossed to a CG1139 CRISPR Knock-in mutant we have 
generated in which the entire CDS region of CG1139 has been deleted and replaced by Gal4 coding sequences (Supplementary Figure 5A). Interestingly, although we observed the expression of CG1139 in some enterocytes and mostly in the posterior midgut region, we also found that cells with a shape characteristic of progenitor cells express CG1139, either in control and infection conditions (Supplementary Figure 5B). This observation suggests that CG1139 may also be important at the level of the progenitor cells and explain the delayed recovery obtained using esg-Gal4 driver. Of note, the knock-in line may have deleted some regulatory elements present in the coding regions or introns and may therefore only partially reflect the endogenous expression of CG1139.

The cytoplasmic purge has previously been shown to be involved in restricting the number of bacteria that cross the epithelium into the hemolymph (Lee et al., 2016). Here, we asked if the knockdown of CG1139 in the gut and the associated delay in the recovery would impact the fitness of the host upon infection. We did not observe any difference in fly locomotion or survival upon infection (Supplementary Figure 6A, B). However, 16h post-infection when $\mathrm{NP}>C G 1139$ RNAi flies still had not fully recovered from the cytoplasmic purge, the loads of SmDb11in these flies were 10-fold higher in the crop and in the midgut compared to control flies (Supplementary Figure 6C). To analyze if this increased bacterial burden resulted from an increased feeding behavior or from constipation caused by $S m \mathrm{Db} 11$ ingestion, we monitored the feeding rate of flies as well as their defecation rate. We did not observe any striking difference for these behaviors upon $S m D b 11$ exposure (Supplementary Figure 6DF), suggesting that the higher bacterial loads are related to the delayed recovery.

CG1139 localizes to the basal part of the gut enterocytes and is required for the retrograde transport

We have shown that a retrograde transport of amino acids is occurring from the hemolymph 
to the gut during the phase of recovery after the cytoplasmic purge. We hypothesized that the amino acid transporter CG1139 might play a role in the retrograde transport, and that it would be required for the fast recovery of the enterocytes upon infection. CG1139 has previously been shown to be localized at the cell surface and on the late endosomal and lysosomal surfaces in Drosophila S2 cells, as well as in the larval fat body. We started by determining the subcellular localization of CG1139 in the midgut of flies by ectopically expressing in enterocytes the functionally active CG1139-GFP fusion. We detected the green fluorescence in the basal part of enterocytes close to the visceral muscles (Figure 3A) in the basal labyrinth region of the cell that allow intensive exchanges of enterocytes with the hemolymph (Shanbhag \& Tripathi, 2009). This basal localization was maintained during infection (Figure 3A), suggesting that CG1139 is likely involved in the amino acid transport from the hemolymph to the gut.

We next asked if the transient increase in the level of free amino acids in the hemolymph $3 \mathrm{~h}$ post-infection would be more pronounced in $N P>C G 1139$ RNAi flies that would reflect their possible accumulation resulting from an impaired importation into the gut epithelium. Unexpectedly, the reverse phenomenon was observed with NP>CG1139 RNAi flies not presenting any increase of free amino acids in the hemolymph $3 \mathrm{~h}$ post-infection, in contrast to the control flies. We next performed the Click-iT AHA technique as described above to analyze if $C G 1139$ would have a role in the retrograde transport after infection. We observed again the retrograde transport to the gut after $S m D b 11$ infection and after starvation in the control flies (Figure 3C). Interestingly, we did not observe the occurrence of a retrograde transport after infection or starvation in $N P>C G 1139$ RNAi flies (Figure 3C), showing that CG1139 is required for the retrograde transport of amino acids into the intestinal epithelium. Altogether, these results suggest that $C G 1139$ is required for the increase of free amino acids in the hemolymph $3 \mathrm{~h}$ post-infection and for their retrograde transport to the gut epithelium. 
Paradoxically, the Click-iT technique is based on a modified methionine that is not expected to be translocated by CG1139, which has been shown to transport alanine, proline, and glycine. These results suggest that CG1139 may act indirectly to support the retrograde transport to the gut epithelium.

Therefore, we decided to determine if CG1139 acts cell-autonomously during the recovery as would be expected for a transporter. Here, we used the escargot Flip Out line (esgF/O) and performed a clonal analysis as previously described (Jiang et al., 2009). The esgF/O flies were crossed with CG1139 RNAi (CG1139 F/O) transgenic flies to generate clones of enterocytes expressing both GFP and RNAi against CG1139 in the offspring. We reasoned that during recovery, two scenarios could take place (Figure 4A): i) both wild type enterocytes and those expressing CG1139 RNAi/GFP would present a similar recovery at 16h post-infection (either growing up to normal size or remaining thin), suggesting a non-cell autonomous role of CG1139; ii) only enterocytes expressing CG1139 RNAi/GFP would remain thin at $16 \mathrm{~h}$, suggesting a cell autonomous role of CG1139. We observed that the first scenario took place, whereby all the enterocytes from the gut recovered to the normal size at $16 \mathrm{~h}$ post-infection (Figure 4B), whether in the CG1139-deficient clones or in wild-type enterocytes. The size of the clones did not influence the results, whether midguts comprised small size clones made-up of a few cells scattered throughout the epithelium or large-size clones of 15-20 cells expressing CG1139 RNAi.

These results show that CG1139 acts non-cell autonomously, confirming the indirect role of CG1139 in the recovery of the enterocytes after the cytoplasmic purge triggered by exposure to $\mathrm{SmDb} 11$. We conclude that CG1139 does not function solely as a regular amino-acid transporter and ensures additional functions in the control of metabolic fluxes between the rest of the organism and the gut epithelium. 


\section{The transcription factor Myc is required for the fast recovery of the gut enterocytes upon}

\section{infection}

To understand how CG1139 functions to ensure the fast recovery, we performed an RNA sequencing analysis in midguts of control flies or flies for which CG1139 was knocked down in the enterocytes. We compared genes differentially expressed at $3 \mathrm{~h}, 8 \mathrm{~h}$, and $16 \mathrm{~h}$ upon SmDb11 infection. We performed a principal component analysis (PCA) and we observed that there was a clear-cut difference on the PCA2 axis between noninfected and infected $N P>$ CG1139 RNAi flies, especially for the $8 \mathrm{~h}$ and $16 \mathrm{~h}$ time-points. This difference was less pronounced between uninfected and infected wild-type control samples (Figure 5A). We also observed that there were consistently more genes differentially expressed in $N P>C G 1139$ RNAi than in the wild-type control samples, when comparing the infected samples to the corresponding sucrose controls (Figure 5B). To analyze which genes would be differentially regulated upon infection between the wild-type and $N P>C G 1139$ RNAi, we performed a Gene Set Enrichment Analysis (GSEA) for all the time-points comparing control and mutant knock-down flies to each other. There were several gene sets that were significantly upregulated in the wild-type compared with the knocked down flies. We focused on the timepoints of $8 \mathrm{~h}$ and $16 \mathrm{~h}$, the period during which the intestinal epithelium recovers its initial shape and thickness. Strikingly, at $8 \mathrm{~h}$ we found an enrichment for genes known to be involved in the positive regulation of organ grown, being up-regulated in the wild-type and not in the $N P>C G 1139$ RNAi flies. In addition, the gene set of lamellipodium assembly was also enriched and up-regulated in the wild-type and not in the $N P>C G 1139$ RNAi (Supplementary Figure 7A). This suggests that there are genes involved in growth, likely required to the increase thickness of the enterocytes, which were not expressed when we knocked down CG1139. Moreover, genes involved in lamellipodium assembly may be involved in actin cytoskeleton, and may be required to close the pore formed during the 
cytoplasmic purge or to re-shape the enterocyte membrane. Alternatively, they might contribute to the formation of microvilli, which are shortened and lost to some extent upon exposure to $\mathrm{SmDb} 11$ hemolysin (Lee et al., 2016). Genes from this category, as well as genes involved in actin nucleation were also up-regulated in the wild-type at 16h (Supplementary Figure 7B). Actin nucleation is the first step in the assembly of the actin filaments, and this may have a similar role described above for the lamellipodium assembly related genes.

To analyze potential candidate genes involved in growth that would be differently regulated between the wild-type and the CG1139 RNAi, we extracted the expression levels of growth related genes from the RNAseq data set and compared infected flies versus their corresponding sucrose control (Figure 5C, Supplementary Figure 8). Interestingly, we noticed that at $16 \mathrm{~h}$ when the enterocytes are still thin in $N P>C G 1139 \mathrm{RNAi}$, these flies downregulated several genes involved with the Insulin signaling pathway, such as Akt1 and InR (Figure 5C). Because CG1139 was reported before to interact with TOR, we tested if the activation of TOR would be different in these flies. We used immunohistology stainings of midguts with antibodies against the phosphorylated form of 4EBP (P-4EBP) as read-out. At 16h after infection, TOR activity was indeed decreased in CG1139 RNAi flies compared to the wild-type controls, in keeping with the possibility that CG1139 contributes to the regulation of TOR activity after infection (Supplementary Figure 9).

We also directly tested the role of the Insulin Receptor gene $\operatorname{In} R$, which was the most downregulated gene in CG1139 RNAi. We knocked down InR in the gut enterocytes using the NP-Gal4 driver. In two independent experiments, we observed either that there was a recovery of the knocked-down $I n R$ flies or alternatively no recovery of the enterocyte thickness, resulting in a bimodal distribution when the two data sets are pooled (Figure 5D). We also focused on the second most down-regulated gene found in CG1139 RNAi flies, which is the gene encoding the MYC transcription factor. This gene is homologous to 
vertebrate $M y c$ proto-oncogenes, is involved in cell growth and it was previously reported to be required for the crypt morphogenesis in the intestine in mice (Bettess et al., 2005). We confirmed that the expression of $M y c$ is significantly downregulated after the ingestion of SmDb11 in NP-Gal4 > CG1139 RNAi compared to control flies at 16h (Supplementary Figure 8B, lmer, $p$-value $=0.0116)$. We decided to further investigate the function of $M y c$ by testing whether knocking down this gene in the gut enterocytes would impair their recovery. While the control flies $w^{1118}$ iso $>$ myc RNAi recovered the normal thickness at $16 \mathrm{~h}$ post infection, NP iso> myc RNAi flies presented a thinner epithelium and were unable to recover the normal size of the gut enterocytes (Figure 5E). These results suggest that $M y c$ expression is downregulated in the CG1139 RNAi flies and that MYC is required for the regrowth of the enterocytes after infection.

Altogether these results indicate that CG1139 seems to regulate the expression of several other genes, such as genes involved in growth, Insulin signaling, and TOR pathway activity. One of these indirect target genes, $M y c$, is also required for the intestinal epithelium recovery after infection. Thus, the relatively reduced expression of $M y c$ in $N P>C G 1139$ RNAi midguts may contribute to their delayed recovery of epithelium thickness.

\section{Discussion}

Ever since the discovery of intestinal stem cells in Drosophila (Micchelli \& Perrimon, 2006;

Ohlstein \& Spradling, 2006) and the interest in intestinal infections (Liehl, Blight, Vodovar, Boccard, \& Lemaitre, 2006; Nehme et al., 2007; Ji-Hwan Ryu et al., 2006), there has been a renewed interest in studying the homeostasis of the intestinal epithelium in normal and 
pathogenic conditions (Nicolas Buchon, Silverman, \& Cherry, 2014; Lemaitre \& MiguelAliaga, 2012; De Navascués et al. 2012; O’Brien et al, 2011). A recent study underlined the importance of the diet in determining the size of the Drosophila gut and emphasized that the adaptation to novel environmental conditions involves not only the proliferation of ISCs but also on enterocyte growth (Bonfini et al., 2021). As TOR signaling contributes to this adaptation, enterocyte growth is implicitly thought to be a cell-autonomous event. Indeed, the mechanisms behind the control of enterocyte growth and regulation of size have gain interest on the field (Xiang et al., 2017; Wen et al., 2017; Tamamouna and Panagi et al, 2020) The enterocyte cytoplasmic purge provides a unique setting in which the regrowth of enterocytes following the extrusion of their apical cytoplasm is uncoupled from ISC proliferation (Lee et al., 2016). Here, we document that the regrowth of enterocytes depends solely on an inversion of metabolic fluxes from the organism to the gut. We demonstrate that the retrograde transport of amino acids involves the CG1139 transporter, which is required for the fast regrowth of enterocytes. Unexpectedly, the function of this transporter is shown genetically to be noncell autonomous.

\section{Retrograde transport of metabolites}

During nutrient uptake, absorption of oligopeptides and amino acids is made through an influx in the apical part of the enterocytes. Once inside the cell, oligopeptides are hydrolyzed by peptidases into their constituent amino acids. While $10 \%$ of these amino acids are used for intracellular protein synthesis, the rest is exported to the internal milieu through an efflux in the basal part of the membrane. The reverse, an influx of amino acids from the blood to the epithelial cells takes place in crypt enterocytes, which do not absorb nutrients from the crypt lumen (Boron \& Boulpaep, 2009). The cytoplasmic purge results in a massive loss of apical cytoplasm for the cells, with a loss of up to $90 \%$ of their total volume in the most severe 
cases. Enterocytes need to recover their shapes fast in order to maintain their normal function. Indeed, recovery happens within the first $16 \mathrm{~h}$ to $20 \mathrm{~h}$ post infection. For this process, it is likely advantageous for the host not to rely on potentially contaminated external food sources, but rather to use internal reserves to reconstitute the organelles and missing cytoplasm and to grow back the enterocytes to their normal size. In starvation conditions, it may be important to maintain enterocyte fitness by the import of amino-acids so as to be able to rapidly digest any food found by the fly. During recovery, we show that the uptake of amino acids in the hemolymph by enterocytes is preceded by a transient increase in their concentration. The retrograde transport of a modified methionine to the gut enterocytes is not observed in the absence of the amino acid transporter CG1139. As recovery is delayed in CG1139 mutant guts, it is likely that the uptake of other amino-acids is also impaired. These amino acids are most likely employed to synthetize new proteins in the cells.

A retrograde transport of amino acids was also observed in flies that were subjected to starvation and sucrose. One explanation may be that under both conditions there is the perception of amino acid starvation, and the organism may need to re-allocate amino acids from the rest of the body to the enterocytes. The gut epithelium of flies exposed to full starvation or amino-acid starvation (sucrose) does not become very thin as when flies as subjected to $S m \mathrm{Db} 11$. This suggests that the retrograde transport of amino acids does not seem to be directly correlated to the size of enterocytes.

\section{Basal localization of CG1139 in the membrane of the gut enterocytes}

We were surprised to detect CG1139 on the baso-lateral side of the midgut epithelium. Indeed, PATs are usually localized to the apical side of the epithelium, where the $\mathrm{pH}$ is lower, thereby transporting metabolites originating from the degradation of dietary nutrients. PAT1 was detected apically in Caco2 cells and in the rat intestine (Anderson et al., 2004; Z. Chen et 
al., 2003) as well as in Aedes aegypti midgut (Evans, Aimanova, \& Gill, 2009). However, an electrophysiology study in the larvae revealed the presence of a H+-ATPase in the basal part of the intestinal epithelium, which establishes a low $\mathrm{pH}$ gradient peaking at 4.34 near the basal labyrinth (Shanbhag \& Tripathi, 2005). The localization of CG1139 in the basal part of the enterocytes is consistent with its putative role in the retrograde transport since CG1139 is a transporter of the SLC36 family of symporters that imports amino-acids concomitantly with a $\mathrm{H}^{+}$proton.

An important question is that of the exact subcellular location of CG1139. PATs such as CG1139 have been described on intracellular compartments such as lysosomes and on the cell surface in Drosophila (Ogmundsdottir et al., 2012). CG1139 may localize both on the cell membrane in the basal labyrinth and on lysosomes where it can interact with the TOR pathway machinery. It will be interesting to characterize its subcellular distribution or redistribution using endosomal/lysosomal markers.

\section{The role of CG1139 in the enterocyte size recovery and its redundancy}

Although we observed that CG1139 is required for the rapid recovery of the normal size of cells after infection, the enterocytes still recover their normal size $48 \mathrm{~h}$ later, in the RNAi knock down flies as well as in the presumably null CRISPR KO mutant. This suggests that other genes are involved in this process, and that this is an integrated response not solely relying on a single gene. The fact that other amino acid transporters were required for the recovery of the gut epithelium leads us to think that they can be acting together in this process and their role may be redundant. While CG1139 belongs to the SLC36 family of protoncoupled amino acid transporters, most of the amino acid transporters that were shown to be required for the recovery belong to the SLC7 family, especially to the cationic amino acid transporters. These transporters are responsible for the major influx of cationic amino acids 
and important for nitric oxide synthesis by delivering L-arginine for nitric oxide synthase (Fotiadis, Kanai, \& Palacín, 2013). Some of the transporters of the SLC7 family have been shown to be involved in growth. Minidisc is required for the growth of imaginal discs in a non-cell autonomous manner (Martin, Hersperger, Simcox, \& Shearn, 2000) and the transporter it encodes has been shown to transport leucine into Insulin Producing Cells (IPCs) in the Drosophila brain, thereby allowing the secretion of insulin-like peptides (Manière, Ziegler, Geillon, Featherstone, \& Grosjean, 2016). Slimfast was shown to regulate organismal growth in the fat body by interacting with TSC/TOR signaling and interfering with PI3-kinase signaling in peripheral tissues. Mutation of slimfast induces systemic growth defects in larvae and leads to smaller adults, similarly to phenotypes observed upon nutrient deprivation or altered TOR signaling (Colombani et al., 2003).

Of note, when we performed the Click-it experiment we established that CG1139 is required for the transport of the labeled methionine analogue from the hemolymph to the gut. However, CG1139 has been shown to transport alanine, proline and glycine in xenopus eggs (Goberdhan et al., 2005). Although we cannot formally exclude the possibility that CG1139 can also transport methionine in vivo, one possibility is that one of the other amino acid transporters identified here to be required for the recovery is transporting the labeled methionine. Although CG1139 was the only amino acid transporter found to be up-regulated during the recovery phase, it will be interesting to further analyze the gene expression of the other amino acid transporters at later time-points. Also, it will be worth determining whether these other amino acid transporters are also localized basally on the cell and required for the retrograde transport of amino acids.

CG1139 and its role in signaling 
Several lines of evidence suggest that the role of $C G 1139$ goes beyond its simple function as an amino acid transporter. In addition to its non-cell autonomous function for the recovery of the gut enterocytes thickness after infection, it was required for the apparition of the peak of amino acids in the hemolymph that is observed in the hemolymph three hours post-infection. This suggests an early role for CG1139 during infection, or that CG1139 establishes a difference in the host physiology a priori, since the knock down is performed for a few days at the adult stage prior to the experimental infection. It will be interesting to determine the origin of these amino acids. They could be generated though the degradation of proteins in muscles or in the fat body, but could also directly originate from hemolymph proteins. Indeed, lipoproteins found in the hemolymph are large proteins, potentially generating a consequent amount of metabolites when degraded.

In addition, CG1139 was also required to modulate the expression of different genes in our RNA sequencing analysis. Of note, genes annotated to be involved in growth in the Gene Ontology analysis were up-regulated in the wild-type during the recovery period. In contrast, some genes involved in Insulin signaling and growth, were more down-regulated in the CG1139 knocked down flies. Myc belongs to a family of proto-oncogenes and was previously shown in Drosophila melanogaster to be required for larval growth and endoreplication (Johnston, Prober, Edgar, Eisenman, \& Gallant, 1999; Pierce et al., 2004). MYC proteins have also been shown to be involved in cell proliferation and differentiation. In mammals, $c$ Myc was shown to be required in the intestinal mucosa for the formation of the correct number of crypts in the small intestine (Bettess et al., 2005). Further rescue experiments will be necessary to assess if $M y c$ is sufficient to rescue the delayed phenotype in CG1139 and if it acts cell autonomously. Also, it will be interesting to explore if the absence of $M y c$ is responsible for a delayed recovery or a complete lack of recovery, also at 48 hours and later. 
CG1139 has previously been shown to interact with TOR pathway and to synergize with its growth promoting effects (Goberdhan et al., 2005; Ogmundsdottir et al., 2012). We also observed lower TOR activity when CG1139 was knocked down in the enterocytes, either after sucrose or $\mathrm{SmDb} 11$ exposure. Indeed, feeding flies with sucrose solution represents an aminoacid starvation condition, which can explain this phenotype. mTORC1 increases ribosome numbers and activity, which promotes the translation of mRNA and stimulates cell growth and proliferation. The main amino acid activator of mTORC1 is leucine, although it can also be activated by glutamine, arginine and serine. CG1139 is not known to transport either of these amino acids. To account for this discrepancy, CG1139 was recently suggested by other group to have a signaling function and to act as a transceptor (Fan \& Goberdhan, 2018). Transceptors have been described as transporter-related receptors and are present in many organisms (Thevelein \& Voordeckers, 2009). Altogether, our data suggest that the role of CG1139 in infection may be signaling to orchestrate the responses necessary for the fast recovery of enterocytes. Moreover, gap junctions or connexin-like coupling was shown to be absent in the posterior midgut enterocytes. (S. R. Shanbhag, Vazhappilly, Sane, D’Silva, \& Tripathi, 2017). Up to now, we have identified genes that are required for recovery in a noncell autonomous manner, suggesting that cell to cell communication may be involved for the' restoration of epithelial thickness.

The size of a given cell type in an organ is usually rather uniform, implying there are inherent processes that regulate the control of final cell size that remain poorly understood at present (Ginzberg, Kafri, \& Kirschner, 2015). There are multiple mechanisms that contribute to the establishment of final cell size including nutrition, biogenesis, and most importantly cell division. Cytoplasmic extrusion as documented here provides a unique setting to study the regulation of cell size independently of most of the parameters cited above. Interestingly, we 
have recently established that the thickness of regrown enterocytes is slightly larger in enterocytes that have been exposed to $S m \mathrm{Db} 11$ hemolysin.

\section{Material and methods}

\section{Fly husbandry}

Drosophila melanogaster flies were raised at $25^{\circ} \mathrm{C}$ and nearly $60 \%$ humidity, $14 \mathrm{~h}$ of daylight and fed a standard semi-solid cornmeal medium (6.4\% (w/v) cornmeal (Moulin des Moines, France), 4.8\% (w/v) granulated sugar (Erstein, France), 1.2\% (w/v) yeast brewer's dry powder (VWR, Belgium), $0.5 \%$ (w/v) agar (Sobigel, France), 0.004\% (w/v) 4hydroxybenzoate sodium salt (Merck, Germany)).

The wild-type flies used for experiments were white wA5001 (Thibault et al., 2004) and the DrosDel $w^{1118}$ isogenic stock ( $w^{1118}$ iso) (Ryder et al., 2004). The following tissue-specific driver lines were used: enterocyte-specific driver line NP1, (also known as Myo31D), NPGal4-tubGal80 (Cronin et al., 2009; Nehme et al., 2007); ubiquitous Ubi-Gal4tubGal80 ${ }^{\text {ts }}$; fat body-specific Yolk-Gal4; enteroendocrine cells-specific prospero-Gal4; midgut progenitorspecific esg-Gal4Gal80 ${ }^{t s}$; visceral muscles-specific how $>$ Gal4; Malpighian tubules-specific uro-Gal4 (X. Li, Rommelaere, Kondo, \& Lemaitre, 2020). CG1139 RNAi used for most of the experiments was the KK line from Vienna Drosophila RNAi Center (VDRC) (CG1139 RNAi), and the same line isogenized to $w^{1118}$ iso (CG1139 RNAi iso) as described in (Ferreira et al., 2014). RNAi lines for the different amino acid transporters were from VDRC. InR RNAi (\#51518) and Myc RNAi (\#51454) were obtained from the Bloomington Stock Center. The drivers were used either alone or crossed with $w^{1118}$ iso as controls, or crossed with a RNAi or a reporter line. Crosses were performed at $25^{\circ} \mathrm{C}$ except for Inr and Myc which were performed at $18^{\circ} \mathrm{C}$. The $\mathrm{F} 1$ progeny of these crosses was then placed at $29^{\circ} \mathrm{C}$ with $70 \%$ humidity for 6 days in order to induce the expression of the Gal4tubGal80 transgenes. The UAS-CG1139-GFP was kindly provided by Prof. Deborah Goberdhan. For clonal analysis, 
the Flp-Out line w; esgGal4tubGal80 ${ }^{t s} U A S-G F P$; UAS-flp Act>CD2>Gal4 from (Jiang et al., 2009) was used. CG1139 knock-out mutant was generated by the Sino-French Hoffmann Institute CRISPR-Cas9 platform and comprises a $5 \mathrm{bp}$ deletion in the sequence causing a presumably truncated non-functional protein (Supplementary Figure 3). CG1139 Knock-in mutant was generated by deleting and replacing the entire CDS region of CG1139 by cassette of Gal4 in Well Genetics (Supplementary Figure 5). Both mutants were isogenized to $w^{1118}$ iso.

\section{Microbiology and Infections}

Serratia marcescens strain Db11 (SmDb11) was cultured on Lysogeny Broth (LB) agar plates with $100 \mu \mathrm{g} / \mathrm{mL}$ of streptomycin. The strain 21C4 Sm21C4 (Kurz et al., 2003) was cultured on LB-agar plates with $20 \mu \mathrm{g} / \mathrm{mL}$ chloramphenicol. The solid plates were placed overnight at $37^{\circ} \mathrm{C}$ to obtain colonies. For liquid cultures, one bacterial colony was taken off the solid plate and inoculated into $200 \mathrm{~mL}$ of liquid LB. These cultures were kept overnight at $37^{\circ} \mathrm{C}$ with agitation. All infections were performed using a final bacterial OD 600 (Optical Density at $600 \mathrm{~nm}$ ) of 10 , except for the survival where $\mathrm{OD}=1$ was used. The OD was measured with a spectrophotometer and $50 \mathrm{~mL}$ of this solution was centrifuged at $4000 \mathrm{~g} / \mathrm{rcf}$ for 10 minutes. The pellet was re-suspended in the appropriate volume of a $50 \mathrm{mM}$ sucrose solution containing $10 \%$ of $\mathrm{LB}$, in order to reach a final $\mathrm{OD}=10.2 \mathrm{~mL}$ of this infection solution were added to two absorbent pads (Millipore AP1003700) that were placed at the bottom of medium-size vials $(3.5 \mathrm{~cm}$ diameter). Twenty female flies of five to seven-day old were fed this infection solution, or sucrose $50 \mathrm{mM}$ as a control at $29^{\circ} \mathrm{C}$.

For Figure 1A, essential amino acids (MEM Amino Acids Solution 50X, Thermo Fischer Scientific \#11130051) were added to the infection solution to a final 1x concentration. The 1x solution is composed of: L-arginine hydrochloride $(126.4 \mathrm{mg} / \mathrm{L})$, L-cysteine $(24 \mathrm{mg} / \mathrm{L})$, L- 
histidine hydrochloride (42 mg/L), L-isoleucine (52.4 mg/L), L-leucine (52.4 mg/L), L-lysine hydrochloride (72.5 mg/L), L-methionine (15.1 mg/L), L-phenylalanine (33 mg/L), Lthreonine (47.6 mg/L), L-tryptophan $(10.2 \mathrm{mg} / \mathrm{L})$, L-tyrosine $(36 \mathrm{mg} / \mathrm{L})$ and L-valine $(46.8$ mg/L). Non-essential amino acids (MEM Non-Essential Amino Acids Solution 100X, Thermo Fischer Scientific \#11140076) were added to a final $1 \mathrm{x}$ concentration. The $1 \mathrm{x}$ solution is composed of: glycine $(15 \mathrm{mg} / \mathrm{L})$, L-alanine $(17.8 \mathrm{mg} / \mathrm{L})$, L-asparagine $(26.4$ mg/L), L-aspartic acid (26.6 mg/L), L-glutamic acid (29.4 mg/L), L-proline (23 mg/L) and Lserine $(21 \mathrm{mg} / \mathrm{L})$.

\section{Fluorescent histochemical staining}

Midguts were dissected in PBS and fixed for 30 minutes with 4\% paraformaldehyde. Samples were washed three times with PBS-Triton X-100 0.1\% (PBT 0.1\%).

\section{Actin staining}

For actin staining midguts were incubated for $1 \mathrm{~h} 30$ at room-temperature or overnight at $4^{\circ} \mathrm{C}$ in $10 \mu \mathrm{M}$ Fluorescein Isothiocyanate (FITC) (Sigma-Aldrich \#P5282) or Texas-Red labeled phalloidin (Invitrogen TM \#T7471). Samples were then washed three times with PBT $0.1 \%$.

\section{GFP staining}

To visualize the CG1139-GFP fusion protein, midguts were incubated with 1:500 anti-GFP (mouse) antibody (Roche \#11814460001) for 3h. Samples were then washed three times with PBT $0.1 \%$ and incubated with a secondary goat anti-mouse FITC antibody (Abcam \#6785) for $1 \mathrm{~h} 30$. In the case of a co-staining with phalloidin and antibodies, the primary antibody was added alone for $3 \mathrm{~h}$, followed by $2 \mathrm{~h}$ of incubation with a mix containing phalloidin and the secondary antibody. The visualization of CG1139 KI> UAS GFP was directly done without antibody staining.

Phospho 4EBP staining 
Midguts were blocked $2 \mathrm{~h}$ in PBT $0.1 \%$ with $2 \%$ bovine serum albumin (BSA). P-4EBP was detected with the anti-phospho-4EBP1 rabbit antibody from Cell Signaling (\#2855). Midguts were incubated for $3 \mathrm{~h}$ with 1:200 of the P-4EBP antibody, washed three times with PBT $0.1 \%$ and incubated with a secondary goat anti-rabbit FITC antibody (Abcam \#6717).

All samples were mounted on diagnostic microscope slides (Thermo Fisher Scientific) with Vectashield plus DAPI (Vector Laboratories). Samples were observed using a LSM780 confocal microscope (Zeiss) or in Axioskop 2 microscope (Zeiss). All images were analyzed with the ImageJ/Fiji software.

\section{Classification of gut epithelium thickness}

To determine the level of thinning and the recovery capacity, we used either qualitative or quantitative analysis. For qualitative analysis, midguts were classified into three different categories according to their epithelial thickness. Thick epithelium, which we represent in blue, corresponds to cells with the normal size, (around $20 \mu \mathrm{m}$ in length), with a clear dome shape characteristic of the intestine. Thin epithelium, which we represent in red, corresponds to cells that are very thin (around $5 \mu \mathrm{m}$ ) and the normal dome shape of the cells is not observed. Semi-thin epithelium, which we represent in yellow, corresponds to cells with intermediate thickness (around $13 \mu \mathrm{m}$ ), where the dome shape is still not completely defined. For quantitative analysis, pictures of midguts were acquired using Fluorescence Axioskope Zeiss and the length of gut enterocytes was measured using ImageJ/Fiji software. The measure for each midgut corresponds to the average of the measure between 10 enterocytes. Measures were taken every 5 to 10 enterocytes. 


\section{$R T q P C R$}

RNA was extracted from 5 or 10 midguts (without crop and Malpighian tubules) in triplicates. Midguts were crushed into $100 \mu \mathrm{L}$ of TRI Reagent RT (Molecular Research Center) with 5\% of bromoanisole (BAN, 98\%, Molecular Research Center). Samples were vortexed, incubated 5 minutes at room temperature and centrifuged 10 minutes, $15000 \mathrm{rcf} / \mathrm{g}$ at $4^{\circ} \mathrm{C}$. The upper phase of the samples was collected, mixed with $350 \mu \mathrm{L}$ of isopropanol and vortexed. Tubes were centrifuged at $18000 \mathrm{rcf} / \mathrm{g}$ for 15 minutes at $4^{\circ} \mathrm{C}$. The pellet was washed twice with 500 $\mu \mathrm{L}$ of ethanol $70 \%$ and dried. RNAs were then re-suspended in $25 \mu \mathrm{L}$ of MilliQ water (Millipore). $1 \mu \mathrm{g}$ of RNA was then used to generate cDNA by reverse transcription, using the iScript cDNA synthesis kit (Bio-Rad \#1708890). The quantitative Polymerase Chain Reaction (qPCR) was performed with the Bio-Rad iQ TM SYBR Green Supermix kit and data were analyzed with the CFX384 system (Bio-Rad). mRNA quantitation was done by normalizing the amount of RNA detected for the gene of interest, with the control rp49 mRNA levels. The relative gene expression was calculated by normalizing the values obtained from sucrose fed flies (controls) versus challenged ones (infected with SmDb11). Primers used for rp49 were the forward 5'GACGCTTCAAGGGACAGTATCTG-3' and the reverse 5'AAACGCGGTTCTGCATGAG3'. Primers used for CG1139 were the forward 5'ACGTCAGCTTTTCGCAGGCCA-3' and the reverse 'ACAACGCAGCCAGGgTGGAC-3'. Primers used for myc were the forward 5' CAGTTCCAGTTCGCAGTCAA-3' and the reverse 5' AGATAAACGCTGCTGGAGGA-3'.

\section{Free amino acids quantification}

Free amino acids were measured using the L-amino acid quantitation kit from Sigma-Aldrich (\#MAK002). Females flies were either starved on sterile water, kept on their normal food, fed sucrose $50 \mathrm{mM}$ or fed the usual infection solution for 3, 6 and $12 \mathrm{~h}$. The hemolymph from 20 
flies or 10 midguts were collected into $10 \mu \mathrm{L}$ of the kit buffer and processed according to the manufacturer's instructions. Samples were run in biological duplicates.

\section{Click-it assay}

The Click-it assay was performed with the Click-it AHA (L-azidohomoalanine) kit from Thermofisher (\#C10102), the Alexa Fluor 488 alkyne dye (\#A10267) and the Click-it Cell Reaction Buffer kit (\#C10269). The reagents were prepared according to the manufacturer's instructions. $50 \mu \mathrm{M}$ of AHA diluted in PBS was injected in the hemolymph of female flies. Flies were placed on different conditions: i) their normal food ii) sterile water iii) sucrose 50 $\mathrm{mM}$ iv) SmDb11 diluted in sucrose 50mM. Midguts were dissected in PBS 6h post-treatment, fixed for 20 minutes in 4\% paraformaldehyde and washed with PBT $0.1 \%$. To detect the injected AHA, midguts were stained for 30 minutes (protected from light) with $0.5 \mathrm{~mL}$ of the following mix: $437.5 \mu \mathrm{L}$ of $1 X$ Click-it Reaction Buffer, $10 \mu \mathrm{L}$ of CuSO 4, $50 \mu \mathrm{L}$ of Click-it Buffer Additive and $2.5 \mu \mathrm{L}$ of $1 \mathrm{mM}$ Alexa Fluor 488 alkyne. Midguts were washed three times with PBT $0.1 \%$ for 15 minutes and mounted on microscopy slides as previously described. Samples were immediately observed at the confocal microscope and the fluorescence intensity was measured with the ImageJ software.

\section{Fitness parameters}

The negative geotaxis assay was performed as described in (Linderman, Chambers, Gupta, \& Schneider, 2012). Ten flies per vial were either infected with an OD600 $=10$ of SmDb11 or fed sucrose as a control. After 3 or $16 \mathrm{~h}$ of feeding, they were transferred in the vials designed 
for the assay without anesthesia. A line was drawn at $8 \mathrm{~cm}$ from the bottom of the tube. The number of flies crossing this line after 5 and 10 seconds was measured and plotted on a graph. Survival assays were performed using 20 flies per vial in triplicates. The experiments were conducted with five to seven-day old adult females at $29^{\circ} \mathrm{C}$ with $70 \%$ humidity. The flies were fed either sucrose $100 \mathrm{mM}$ as a control or infected with an OD600=1 of SmDb11. Two absorbent pads were placed at the bottom of medium-size vials and $2 \mathrm{~mL}$ of sucrose or bacterial solution were added to the filters. Each day, flies that were alive were counted and $200 \mu \mathrm{L}$ of $100 \mathrm{mM}$ sucrose was added to the vials.

Bacterial loads in the midguts or crop were accessed $16 \mathrm{~h}$ post SmDb11 infection. Guts were dissected and pools of 3 midguts and crops were homogenized in $100 \mu 1$ of PBS1x and serial dilutions were performed to plate. Extracts were plated in LB agar containing $100 \mu \mathrm{g} / \mathrm{mL}$ of streptomycin and incubated at $37^{\circ} \mathrm{C}$ overnight to count total number of colonies.

Food intake was accessed either by quantifying the level of ingested blue dye or using the FLIC system. To access the level of ingested blue dye, flies were either fed in sucrose or SmDb11, in a solution containing $5 \%$ of standard food blue dye. After $16 \mathrm{~h}$ females were homogenized in $50 \mu \mathrm{m}$ of PBS1x and samples were centrifuged at maximum speed for 10min. Absorbance was accessed using the Varioscan and the wavelength was measured at $625 \mathrm{~nm}$. The absorbance for each sample was calculated to the relative controls, where flies were subjected to the same conditions but without blue dye. Each sample corresponds to a pool of 10 females. To access the food intake using the FLIC system (Ro, Harvanek, \& Pletcher, 2014) we placed one fly per well feeding either in sucrose or SmDb11 solution. 12 females were accessed per condition in each experimental replicate. Number of leaks was considered as a read-out for the food intake.

Defecation was accessed by feeding 10 females per vial either in sucrose or in $S m \mathrm{Db} 11$, in a solution containing $5 \%$ of standard food blue dye. Fecal spots on the vial walls were counted. 


\section{$R N A$ sequencing}

To analyze genes differentially regulated in NP> ctrl and NP> CG1139 RNAi flies after infections, flies were infected with SmDb11 or exposed to sucrose and midguts were dissected $3 \mathrm{~h}, 8 \mathrm{~h}$, or $12 \mathrm{~h}$ post infection. Each sample comprised 10 midguts (without crop and Malpighian tubules) and three replicate samples were generated per condition.

Samples were sent to BGI and RNA was sequenced using the DNBseq platform. We used the Agilent 2100 Bio analyzer (Agilent RNA 6000 Nano Kit) to perform the total RNA sample quality control RNA concentration, 28S/18S, and the fragment length distribution. Firstly, we removed the reads mapped to rRNAs and obtained raw data; then, we filtered out the low quality reads (More than $20 \%$ of the bases qualities are lower than 10), reads with adaptors and reads with unknown bases ( $\mathrm{N}$ bases more than 5\%) to get the clean reads. We assembled those clean reads into Unigenes, followed with Unigene functional annotation and calculated the Unigene expression levels and SNPs of each sample. Finally, we identify DEGs (differential expressed genes) between samples and performed clustering analysis and functional annotations. After filtering the reads, clean reads were mapped to reference genome using HISAT2 (Kim, Langmead, \& Salzberg, 2015) and the average mapping ratio with reference genome was $94.08 \%$. Clean reads were mapped to reference transcripts using Bowtie2 (Langmead \& Salzberg, 2012), and gene expression level was calculated for each sample with RSEM (B. Li \& Dewey, 2011). Based on the gene expression level, we used DEseq2 algorithms to identify the DEG (Differentially expression genes) between samples or groups. With DEGs we performed Gene Ontology (GO) classification, KEGG pathway classification and functional enrichment. We calculated false discovery rate (FDR) for each pvalue, in general, the terms which FDR not larger than 0.01 are defined as significant 
enriched. We extracted here genes involved in cell growth where we identified genes involved in insulin signaling and the transcription factor myc.

The average mapping ratio with reference genome was $94.08 \%$, the average mapping ratio with gene was $80.06 \%$ and a total of 17,003 genes were detected.

Gene Set Enrichment Analysis (GSEA) was performed as described (Subramanian et al., 2005) using GSEA v4.1.0. We used a gene set library from the FlyEnrichr (E. Y. Chen et al., 2013; Kuleshov et al., 2016) that distributes the genes according to biological processes from their Gene Ontology, “GO_Biological_Process_AutoRIF_Predicted_zscore”.

\section{Statistical analysis}

Statistical analyses were performed using GraphPad software Prism 6 and R. For qualitative analysis of epithelium thickness, we used chi-square statistical tests. We also used Linear models (lm), or linear mixed-effect models (lmer) (Bates, Mächler, Bolker, \& Walker, 2015)(lmer package lme4) if there were random factors. Significance of interactions between factors was tested by comparing models fitting the data with and without the interactions using analysis of variance (anova). Models were simplified when interactions were not significant. Pairwise comparisons of the estimates from fitted models were analyzed using lmerTest, lsmean, and multcomp packages.

\section{Acknowledgements}

We thank Prof. Deborah Goberdhan to have kindly provided the UAS-CG1139-GFP line, the Vienna Drosophila Resource Center (VDRC, www.vdrc.at) and Bloomington Drosophila Stock Center (NIH P40OD018537) for their resource. We are grateful to Prof. Jiyong Lu and the SFHI CRISPR-Cas9 platform for generating the CG1139 KO strain. 
We thank Jenny Nguyen for expert technical help in some experiments and to Roenick Olmo for the help with the GSEA analysis.

This work has been funded by CNRS, University of Strasbourg, ANR grant ANR-16-CE130011-01 (ENTEROCYTE_PURGE_RECOVERY), Fondation pour la Recherche Médicale (Equipe FRM DEQ20090515394 to DF) and fellowship FDT20170437224 to CS. Our work is also partially sponsored by Infinitus, Inc (China). The funding sources had no role in the design of the study nor in its execution, analyses, interpretation of the data or decision to publish the results. 


\section{Bibliography}

Anderson, C. M. H., Grenade, D. S., Boll, M., Foltz, M., Wake, K. A., Kennedy, D. J., ... Thwaites, D. T. (2004). H+/amino acid transporter 1 (PAT1) is the imino acid carrier: An intestinal nutrient/drug transporter in human and rat. Gastroenterology, 127(5), 1410-1422. https://doi.org/10.1053/j.gastro.2004.08.017

Apidianakis, Y., Pitsouli, C., Perrimon, N., \& Rahme, L. (2009). Synergy between bacterial infection and genetic predisposition in intestinal dysplasia. Proceedings of the National Academy of Sciences of the United States of America, 106(49), 20883-20888. https://doi.org/10.1073/pnas.0911797106

Bates, D., Mächler, M., Bolker, B. M., \& Walker, S. C. (2015). Fitting linear mixed-effects models using lme4. Journal of Statistical Software, 67(1). https://doi.org/10.18637/jss.v067.i01

Bettess, M. D., Dubois, N., Murphy, M. J., Dubey, C., Roger, C., Robine, S., \& Trumpp, A. (2005). c-Myc Is Required for the Formation of Intestinal Crypts but Dispensable for Homeostasis of the Adult Intestinal Epithelium. Molecular and Cellular Biology, 25(17), 7868-7878. https://doi.org/10.1128/mcb.25.17.7868-7878.2005

Bonfini, A., Dobson, A. J., Duneau, D., Revah, J., Liu, X., Houtz, P., \& Buchon, N. (2021). Multiscale analysis reveals that diet-dependent midgut plasticity emerges from alterations in both stem cell niche coupling and enterocyte size. ELife, 10, 1-41. https://doi.org/10.7554/elife.64125

Bonfini, A., Liu, X., \& Buchon, N. (2016). From pathogens to microbiota: How Drosophila intestinal stem cells react to gut microbes. Developmental and Comparative Immunology, 64, 22-38. https://doi.org/10.1016/j.dci.2016.02.008

Boron, W. F., \& Boulpaep, E. L. (2009). Medical physiology: A cellular and molecular approach (2nd ed., International ed.). Philadelphia, PA: Saunders/Elsevier.

Buchon, N, Broderick, N. A., Chakrabarti, S., \& Lemaitre, B. (2009). Invasive and indigenous microbiota impact intestinal stem cell activity through multiple pathways in Drosophila. Genes \& Development, Vol. 23, pp. 23332344.

Buchon, Nicolas, Silverman, N., \& Cherry, S. (2014). Immunity in Drosophila melanogaster — from microbial recognition to whole-organism physiology. Nature Reviews Immunology, 14(12), 796-810. https://doi.org/10.1038/nri3763

Chen, E. Y., Tan, C. M., Kou, Y., Duan, Q., Wang, Z., Meirelles, G. V., ... Ma'ayan, A. (2013). Enrichr: interactive and collaborative HTML5 gene list enrichment analysis tool. BMC Bioinformatics, 157(14), 617-619. https://doi.org/10.1007/s00701-014-2321-4

Chen, Z., Fei., Y. J., Anderson, C. M. H., Wake, K. A., Miyauchi, S., Huang, W., ... Ganapathy, V. (2003). Structure, function and immunolocalization of a proton-coupled amino acid transporter (hPAT1) in the human intestinal cell line Caco-2. Journal of Physiology, 546(2), 349-361. https://doi.org/10.1113/jphysiol.2002.026500

Colombani, J., Raisin, S., Pantalacci, S., Radimerski, T., Montagne, J., \& Léopold, P. (2003). A nutrient sensor mechanism controls Drosophila growth. Cell, 114(6), 739-749. https://doi.org/10.1016/S0092-8674(03)00713-X

Cronin, S. J. F., Nehme, N. T., Limmer, S., Liegeois, S., Pospisilik, J. A., Schramek, D., ... Penninger, J. M. (2009). GenomeWide RNAi Screen Identifies Pathogenic Bacterial Infection. Science, 325(July), 340-344.

De Navascués, J., Perdigoto, C. N., Bian, Y., Schneider, M. H., Bardin, A. J., Martínez-Arias, A., \& Simons, B. D. (2012). Drosophila midgut homeostasis involves neutral competition between symmetrically dividing intestinal stem cells. EMBO Journal, 31(11), 2473-2485. https://doi.org/10.1038/emboj.2012.106

Evans, A. M., Aimanova, K. G., \& Gill, S. S. (2009). Characterization of a blood-meal-responsive proton-dependent amino acid transporter in the disease vector, Aedes aegypti. Journal of Experimental Biology, 212(20), 32633271. https://doi.org/10.1242/jeb.029553

Fan, S. J., \& Goberdhan, D. C. I. (2018). PATs and SNATs: Amino acid sensors in disguise. Frontiers in Pharmacology, 9(JUN), 1-8. https://doi.org/10.3389/fphar.2018.00640

Featherstone, D. E. (2011). Glial solute carrier transporters in drosophila and mice. Glia, 59(9), 1351-1363. https://doi.org/10.1002/glia.21085

Ferrandon, D. (2013). The complementary facets of epithelial host defenses in the genetic model organism Drosophila melanogaster: From resistance to resilience. Current Opinion in Immunology, 25(1), 59-70. https://doi.org/10.1016/j.coi.2012.11.008

Ferreira, A. G., Naylor, H., Esteves, S. S., Pais, I. S., Martins, N. E., \& Teixeira, L. (2014). The Toll-Dorsal Pathway Is Required for Resistance to Viral Oral Infection in Drosophila. PLoS Pathogens, 10(12), e1004507. https://doi.org/10.1371/journal.ppat.1004507

Flint, H. J., Scott, K. P., Louis, P., \& Duncan, S. H. (2012). The role of the gut microbiota in nutrition and health. Nature Reviews Gastroenterology \& Hepatology, 9(10), 577-589. https://doi.org/10.1038/nrgastro.2012.156

Fotiadis, D., Kanai, Y., \& Palacín, M. (2013). The SLC3 and SLC7 families of amino acid transporters. Molecular Aspects of Medicine, 34(2-3), 139-158. https://doi.org/10.1016/j.mam.2012.10.007

Ginzberg, M. B., Kafri, R., \& Kirschner, M. (2015). On being the right (cell) size. Science, 348(6236). 
https://doi.org/10.1126/science.1245075

Goberdhan, D. C. I., Meredith, D., Boyd, C. A. R., \& Wilson, C. (2005). PAT-related amino acid transporters regulate growth via a novel mechanism that does not require bulk transport of amino acids. (June). https://doi.org/10.1242/dev.01821

Jiang, H., Patel, P. H., Kohlmaier, A., Grenley, M. O., McEwen, D. G., \& Edgar, B. A. (2009). Cytokine/Jak/Stat Signaling Mediates Regeneration and Homeostasis in the Drosophila Midgut. Cell, 137(7), 1343-1355. https://doi.org/10.1016/j.cell.2009.05.014

Johnston, L. A., Prober, D. A., Edgar, B. A., Eisenman, R. N., \& Gallant, P. (1999). Drosophila myc regulates cellular growth during development. Cell, 98(6), 779-790. https://doi.org/10.1016/S0092-8674(00)81512-3

Kim, D., Langmead, B., \& Salzberg, S. L. (2015). HISAT: A fast spliced aligner with low memory requirements. Nature Methods, 12(4), 357-360. https://doi.org/10.1038/nmeth.3317

Kuleshov, M. V., Jones, M. R., Rouillard, A. D., Fernandez, N. F., Duan, Q., Wang, Z., ... Ma'ayan, A. (2016). Enrichr: a comprehensive gene set enrichment analysis web server 2016 update. Nucleic Acids Research, 44(W1), W90W97. https://doi.org/10.1093/nar/gkw377

Kurz, Â., Chauvet, S., Le, C., Aurouze, M., Vallet, I., Bentzmann, S. De, ... Ewbank, J. J. (2003). Virulence factors of the human opportunistic pathogen Serratia marcescens identified by in vivo screening. 22(7), 1451-1460.

Kurz, C. L., Chauvet, S., Andrès, E., Aurouze, M., Vallet, I., Michel, G. P. F., ... Ewbank, J. J. (2003). Virulence factors of the human opportunistic pathogen Serratia marcescens identified by in vivo screening. EMBO Journal, 22(7), 14511460. https://doi.org/10.1093/emboj/cdg159

Langmead, B., \& Salzberg, S. L. (2012). Fast gapped-read alignment with Bowtie 2. Nature Methods, 9(4), 357-359. https://doi.org/10.1038/nmeth.1923

Lee, K., Lestradet, M., Socha, C., Simon-assmann, P., Ferrandon, D., Lee, K., ... Schmitz, A. (2016). Enterocyte Purge and Rapid Recovery Is a Resilience Reaction of the Gut Epithelium to Pore-Forming Toxin Attack. Cell Host \& Microbe, 20(December), 716-730. https://doi.org/10.1016/j.chom.2016.10.010

Lemaitre, B., \& Miguel-Aliaga, I. (2012). The Digestive Tract of Drosophila melanogaster. Annu. Rev. Genet., Vol. 47, p. 130909162859001.

Li, B., \& Dewey, C. N. (2011). RSEM: accurate transcript quantification from RNA-Seq data with or without a reference genome Bo. BMC Bioinformatics, 21-40. https://doi.org/10.1201/b16589

Li, X., Rommelaere, S., Kondo, S., \& Lemaitre, B. (2020). Renal Purge of Hemolymphatic Lipids Prevents the Accumulation of ROS-Induced Inflammatory Oxidized Lipids and Protects Drosophila from Tissue Damage. Immunity, 52(2), 374-387.e6. https://doi.org/10.1016/j.immuni.2020.01.008

Liehl, P., Blight, M., Vodovar, N., Boccard, F., \& Lemaitre, B. (2006). Prevalence of Local Immune Response against Oral Infection in a Drosophila/Pseudomonas Infection Model. PLoS Pathog, Vol. 2, p. e56.

Linderman, J. A., Chambers, M. C., Gupta, A. S., \& Schneider, D. S. (2012). Infection-Related Declines in Chill Coma Recovery and Negative Geotaxis in Drosophila melanogaster. PLoS ONE, 7(9). https://doi.org/10.1371/journal.pone.0041907

Manière, G., Ziegler, A. B., Geillon, F., Featherstone, D. E., \& Grosjean, Y. (2016). Direct Sensing of Nutrients via a LAT1like Transporter in Drosophila Insulin-Producing Cells. Cell Reports, 17(1), 137-148. https://doi.org/10.1016/j.celrep.2016.08.093

Martin, J. F., Hersperger, E., Simcox, A., \& Shearn, A. (2000). Minidiscs Encodes a Putative Amino Acid Transporter Subunit Required Non-Autonomously for Imaginal Cell Proliferation. Mechanisms of Development, 92(2), 155167. https://doi.org/10.1016/S0925-4773(99)00338-X

Micchelli, C. A., \& Perrimon, N. (2006). Evidence that stem cells reside in the adult Drosophila midgut epithelium. Nature, 439(7075), 475-479. https://doi.org/10.1038/nature04371

Nehme, N. T., Li??geois, S., Kele, B., Giammarinaro, P., Pradel, E., Hoffmann, J. A., ... Ferrandon, D. (2007). A model of bacterial intestinal infections in Drosophila melanogaster. PLoS Pathogens, 3(11), 1694-1709. https://doi.org/10.1371/journal.ppat.0030173

O'Brien, L. E., Soliman, S. S., Li, X., \& Bilder, D. (2011). Altered Modes of Stem Cell Division Drive Adaptive Intestinal Growth. Cell, Vol. 147, pp. 603-614.

Ogmundsdottir, M., Heublein, S., Kazi, S., Reynolds, B., Visvalingam, S. M., Shaw, M. K., \& Goberdhan, D. C. I. (2012). Proton-Assisted Amino Acid Transporter PAT1 Complexes with Rag GTPases and Activates TORC1 on Late Endosomal and Lysosomal Membranes. 7(5). https://doi.org/10.1371/journal.pone.0036616

Ohlstein, B., \& Spradling, A. (2006). The adult Drosophila posterior midgut is maintained by pluripotent stem cells. Nature, 439(7075), 470-474. https://doi.org/10.1038/nature04333

Pierce, S. B., Yost, C., Britton, J. S., Loo, L. W. M., Flynn, E. M., Edgar, B. A., \& Eisenman, R. N. (2004). dMyc is required for larval growth and endoreplication in Drosophila. Development, 131(10), 2317-2327. https://doi.org/10.1242/dev.01108

Ro, J., Harvanek, Z. M., \& Pletcher, S. D. (2014). FLIC: High-throughput, continuous analysis of feeding behaviors in 
bioRxiv preprint doi: https://doi.org/10.1101/2021.10.29.466403; this version posted October 30, 2021. The copyright holder for this preprint (which was not certified by peer review) is the author/funder. All rights reserved. No reuse allowed without permission.

Drosophila. PLoS ONE, 9(6). https://doi.org/10.1371/journal.pone.0101107

Ryder, E., Blows, F., Ashburner, M., Bautista-Llacer, R., Coulson, D., Drummond, J., ... Russell, S. (2004). The DrosDel collection: A set of P-element insertions for generating custom chromosomal aberrations in Drosophila melanogaster. Genetics, 167(2), 797-813. https://doi.org/10.1534/genetics.104.026658

Ryu, J.-H, Kim, S.-H., Lee, H.-Y., Bai, J. Y., Nam, Y.-D., Bae, J.-W., ... Lee, W.-J. (2008). Innate Immune Homeostasis by the Homeobox Gene Caudal and Commensal-Gut Mutualism in Drosophila. Science, Vol. 319, pp. 777-782.

Ryu, Ji-Hwan, Ha, E.-M., Oh, C.-T., Seol, J.-H., Brey, P. T., Jin, I., ... Lee, W.-J. (2006). An essential complementary role of NF- $\kappa$ B pathway to microbicidal oxidants in Drosophila gut immunity. EMBO J, Vol. 25, pp. 3693-3701.

Shanbhag, S. R., Vazhappilly, A. T., Sane, A., D’Silva, N. M., \& Tripathi, S. (2017). Electrolyte transport pathways induced in the midgut epithelium of Drosophila melanogaster larvae by commensal gut microbiota and pathogens. Journal of Physiology, 595(2), 523-539. https://doi.org/10.1113/JP272617

Shanbhag, S., \& Tripathi, S. (2005). Electrogenic H+ transport and pH gradients generated by a V-H+-ATPase in the isolated perfused larval Drosophila midgut. Journal of Membrane Biology, 206(1), 61-76. https://doi.org/10.1007/s00232-005-0774-1

Shanbhag, Shubha, \& Tripathi, S. (2009). Epithelial ultrastructure and cellular mechanisms of acid and base transport in the Drosophila midgut. Journal of Experimental Biology, 212(11), 1731-1744. https://doi.org/10.1242/jeb.029306

Subramanian, A., Tamayo, P., Mootha, V. K., Mukherjee, S., Ebert, B. L., Gillette, M. A., ... Mesirov, J. P. (2005). Gene set enrichment analysis: A knowledge-based approach for interpreting genome-wide expression profiles. Proceedings of the National Academy of Sciences of the United States of America, 102(43), 15545-15550. https://doi.org/10.1073/pnas.0506580102

Tamamouna, V., Panagi, M., Theophanous, A., Demosthenous, M., Michail, M., Papadopoulou, M., ... Apidianakis, Y. (2020). Evidence of two types of balance between stem cell mitosis and enterocyte nucleus growth in the Drosophila midgut. Development (Cambridge, England), 147(11), 1-14. https://doi.org/10.1242/dev.189472

Thevelein, J. M., \& Voordeckers, K. (2009). Functioning and evolutionary significance of nutrient transceptors. Molecular Biology and Evolution, 26(11), 2407-2414. https://doi.org/10.1093/molbev/msp168

Thibault, S. T., Singer, M. A., Miyazaki, W. Y., Milash, B., Dompe, N. A., Singh, C. M., ... Margolis, J. (2004). A complementary transposon tool kit for Drosophila melanogaster using P and piggyBac. Nature Genetics, 36(3), 283-287. https://doi.org/10.1038/ng1314

Thwaites, D. T., \& Anderson, C. M. H. (2011). The SLC36 family of proton-coupled amino acid transporters and their potential role in drug transport. British Journal of Pharmacology, 164(7), 1802-1816. https://doi.org/10.1111/j.1476-5381.2011.01438.x

Wen, J. K., Wang, Y. T., Chan, C. C., Hsieh, C. W., Liao, H. M., Hung, C. C., \& Chen, G. C. (2017). Atg9 antagonizes TOR signaling to regulate intestinal cell growth and epithelial homeostasis in Drosophila. ELife, 6, 1-22. https://doi.org/10.7554/eLife.29338

Xiang, J., Bandura, J., Zhang, P., Jin, Y., Reuter, H., \& Edgar, B. A. (2017). EGFR-dependent TOR-independent endocycles support Drosophila gut epithelial regeneration. Nature Communications, 8(May), 1-13. https://doi.org/10.1038/ncomms15125

Zhu, G., Deng, J., \& Yin, Y. (2018). Amino Acids As Mediators of Metabolic Cross Talk between Host and Pathogen. 9(February). https://doi.org/10.3389/fimmu.2018.00319 


\section{Figures Legend}

\section{Figure 1. - Recovery of enterocytes after infection entails retrograde transport from}

body reserves. (A) Flies were infected in a sucrose solution containing 10\% LB medium, PBS1x or a mix of essential and non-essential amino-acids (AA). Thickness of gut epithelium was accessed at $3 \mathrm{~h}$ and $16 \mathrm{~h}$ after $S m \mathrm{Db} 11$ infection. All conditions recover to a thick epithelium $16 \mathrm{~h}$ post infection. (B) Flies were infected for $3 \mathrm{~h}$ with $S m \mathrm{Db} 11$ suspended in PBS1x solution, and then transferred to sucrose with $10 \%$ of LB medium solution, PBS1x or to $\mathrm{H} 2 \mathrm{O}$. Thickness of gut epithelium was accessed at $3 \mathrm{~h}$ after infection and at $13 \mathrm{~h}$ after transferring the flies to the second solution. Guts were stained with phalloidin and representative pictures of gut enterocytes from each condition are shown in (C). (D) Flies fed in standard food or flies starved for $24 \mathrm{~h}$ were infected with $S m \mathrm{Db} 11$ and thickness of gut epithelium was accessed at $16 \mathrm{~h}$ post infection. $16 \mathrm{~h}$ post infection the percentage of midguts with thick epithelia is lower when flies are pre-starved. Representative pictures of gut enterocytes are shown in (E). (F) Quantification of free amino-acids in the hemolymph of flies $3 \mathrm{~h}$ and $6 \mathrm{~h}$ after sucrose exposure or $\mathrm{SmDb} 11$ infection. The level of free amino-acids increases in the hemolymph at 6h (One-way ANOVA, $p<0.001)$. (G) Click-it AHA kit was used to assess a retrograde transport from hemolymph to the intestine. Flies were injected with $50 \mu \mathrm{M}$ of AHA and placed on different conditions: regular food, infected with $S m \mathrm{Db} 11$, sucrose $50 \mathrm{mM}$ or water (starved). Midguts were dissected 6h later, and stained with the alkyne probe to assess the fluorescence corresponding to the incorporated amino-acid. Detailed scheme in Supplementary figure 1B. Fluorescence was calculated by measuring green (Alexa 488) intensity with the Image $\mathrm{J}$ software. The increase in fluorescence was considered as read-out for the level of retrograde transport from the midgut. Infected flies 
presented the higher level of retrograde transported (One-way ANOVA; $*=\mathrm{p}<0,05$; $* * * *=\mathrm{p}<0,0001)$

Green $=$ Actin. Qualitative quantification of intestines according to their epithelial thickness : thin (red), semithin (yellow) or thick (blue).

\section{Figure 2. Fast recovery of gut epithelial thickness requires the CG1139 amino acid}

transporter. (A) Genes induced in the midgut during infection with $\operatorname{SmDb} 11$ identified by RNA-sequencing. RNA was extracted from 10 midguts in triplicates. 144, 355 and 176 genes are induced 3, 6 and 9h post-infection in the intestine, respectively, and 92 genes are induced throughout the infection. (B) CG1139 expression measured by RT-qPCR. RNA was extracted from 10 midguts, in triplicates. Bars represent expression of infected flies relative to sucrose controls. CG1139 was induced in the midgut during the recovery, 9h post-infection with SmDb11 (One-way ANOVA, $* * * *=p \quad<0,0001)$ (C) Control flies $(N P>c t r l)$ and $N P>C G 1139-\mathrm{RNAi}$ were infected with $\mathrm{SmDb} 11$, midguts were dissected at $3 \mathrm{~h}, 16 \mathrm{~h}$, and $48 \mathrm{~h}$ post infection and the thickness of the gut epithelium was accessed. Midguts from $N P>C G 1139-$ RNAi flies are thinner than the control 16h post-infection. (D) Representative midguts from control flies and $N P>C G 1139-$ RNAi flies 16h post-infection. Blue: DNA stained with DAPI; Red: actin stained with phalloidin. (E) Epithelium thickness $16 \mathrm{~h}$ after SmDb11 infection in $N P>\operatorname{ctrl}, N P>C G 1139-\mathrm{RNAi}$ and in the rescued line $N P>C G 1139$ RNAi;UAS-CG1139-GFP. The rescue of CG1139 rescues the delay on epithelium recovery. (F) NP> $w^{1118}$ iso and NP>CG1139 RNAi iso were exposed to sucrose or infected with SmDb11 and enterocytes thickness was measured using ImageJ software. Each dot represents the average of 10 independent measures in one midgut. $N P>w^{1118}$ iso recover the normal size of the enterocytes thickness compared to sucrose, but enterocytes from $N P>C G 1139$ RNAi iso remain thin $16 \mathrm{~h}$ post infection $(\operatorname{lmer}, * * *=p<0.001)$. (G) $w^{1118}$ iso and a Knock-out 
CRISPR mutant for CG1139 (CG1139 iso CRISPR KO) were exposed to sucrose (Not infected) or infected with $S m D b 11$ and enterocytes thickness was measured as in (F). CG1139 mutant has a delay in recovery at $16 \mathrm{~h}$ but recovers the normal enterocyte thickness $48 \mathrm{~h}$ post infection similar to sucrose condition (lmer, $* * *=p<0.001)$. (H) Flies where CG1139 was knocked down in the enterocytes (NP>CG1139 RNAi iso) or in the ISCs (esg>CG1139 RNAi iso) and the respective controls $\left(N P>w^{1118}\right.$ iso and $e s g>w^{1118}$ iso $)$ were infected with SmDb11 and midguts were dissected $3 \mathrm{~h}$ and $16 \mathrm{~h}$ post infection to assess the epithelium thickness. Both NP>CG1139 RNAi iso and esg $>$ CG1139 RNAi iso present a delay in recovery, with higher percentage of midguts with thin epithelium $16 \mathrm{~h}$ post infection compared to the respective controls.

Quantification of intestines according to their epithelial thickness: thin (red), semithin (yellow) or thick (blue).

Figure 3. CG1139 localizes to the basal side of the enterocytes in the midgut epithelium and is required for the retrograde transport (A) Confocal pictures of dissected midguts from $N P>U A S-C G 1139-G F P$ flies exposed to sucrose (Non-infected), or exposed to $S m \mathrm{Db} 11$ for 3h or 6h. Blue: DNA; Red: actin; Green: CG1139 GFP. (B) Quantification of free aminoacids in the hemolymph of $N P>\operatorname{ctrl}$ and $N P>C G 1139$ RNAi $3 \mathrm{~h}$ after sucrose exposure or SmDb11 infection. The level of free amino-acids increases in the hemolymph of $N P>$ ctrl flies but not in NP>CG1139 RNAi flies (One-way ANOVA, *** $=p<0.001)$. (C) Click-it AHA kit was used to assess a retrograde transport from hemolymph to the intestine as described in Figure 1. There is an increase of retrograde transport to the midgut in $N P>\operatorname{ctrl}$ flies after infection or starvation but not in NP>CG1139 RNAi. (One-way ANOVA, **** $=p$ $<0.0001)$ 
Figure 4. CG1139 is required non-cell autonomously for the recovery of the intestinal epithelium after SmDb11 infection. Clonal analyses were performed using the escargot Flp Out (esgF/O) system. (A) w; esgGal4tubGal80ts UAS-GFP; UAS-flp Act $>C D 2>$ Gal4 flies were crossed to UAS-CG1139-RNAi flies to generate clones of enterocytes expressing both GFP and RNAi against CG1139 (F/O CG1139). Left scenario: both RNAi and wild type enterocyte recover or stay thin $16 \mathrm{~h}$ post-infection, suggesting a non cell-autonomous mechanism. Right scenario: RNAi enterocytes remain thin and wild-type enterocytes recover, suggesting a cell-autonomous mechanism of CG1139. (B) Confocal pictures of dissected midguts from control F/O or CG1139 F/O flies. Blue: DNA; Red: Actin; Green: control GFP clone or CG1139-RNAi GFP clone. In F/O control and F/O CG1139 flies, both RNAi and wild type enterocytes showed the same recovery rate $16 \mathrm{~h}$ post-infection, suggesting a non cell-autonomous mechanism.

Figure 5. The transcription factor $M y c$ is required for the recovery of the gut enterocytes after infection. $N P>\operatorname{ctrl}$ and $N P>$ CG1139 RNAi were exposed to sucrose or infected with $S m \mathrm{Db} 11$ for $3 \mathrm{~h}, 8 \mathrm{~h}$ and $16 \mathrm{~h}$. RNA sequencing was performed in dissected midguts. (A) Principal component analyses for gene expression of different samples. (B) Venn diagram for each time-point comparing genes differentially expressed in $N P>$ ctrl and $N P>C G 1139$ RNAi after infection, relative to sucrose. (C) Genes involved in growth that are up- or down-regulated in CG1139 RNAi 16h after infection (relative to sucrose). Insulin related genes and the growth transcription factor $M y c$ are down-regulated in $N P>C G 1139$ RNAi. (D) InR and (E) Myc were knocked down in the gut enterocytes (NPiso $>$ Inr RNAi and NPiso $>$ Myc RNAi) and compared with the respective controls ( $w^{1118}$ iso $>$ Inr RNAi and $w^{1118}$ iso $>$ Myc RNAi) for the recovery of the gut enterocytes thickness $16 \mathrm{~h}$ upon infection or exposure to sucrose. All the flies present a thin epithelium at $3 \mathrm{~h}$ upon infection. While the 
thickness of the gut enterocytes in NPiso $>$ Inr RNAi $16 \mathrm{~h}$ post infection are not significantly different from the sucrose control, NPiso $>$ Myc RNAi are not able to recover the normal enterocyte thickness compared to the sucrose control $($ lmer, $* * *=p<0.001)$. 


\section{Figure 1}

A

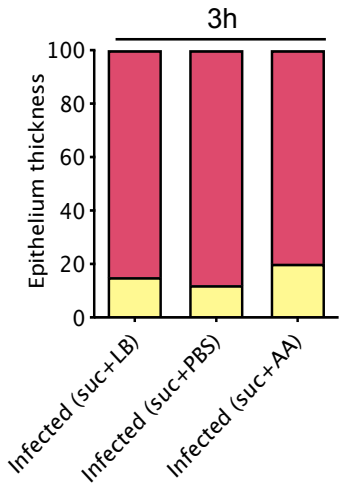

C

Infected $3 \mathrm{~h}$

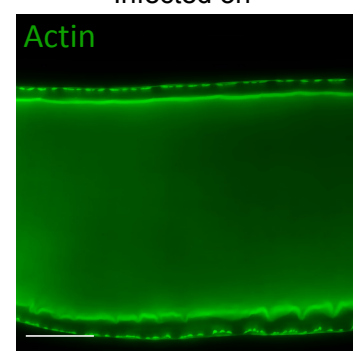

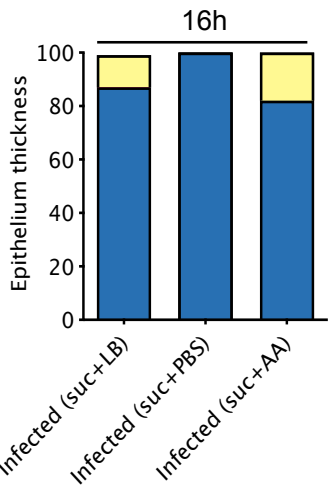

B

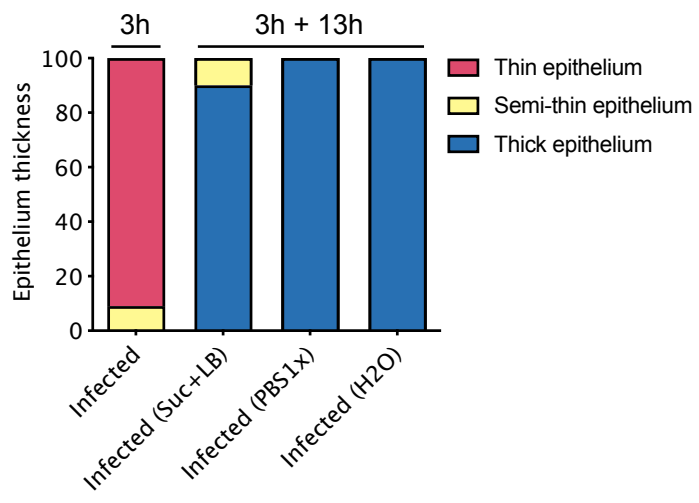

Infected $3 \mathrm{~h} \rightarrow 13 \mathrm{~h}$ Suc + LB
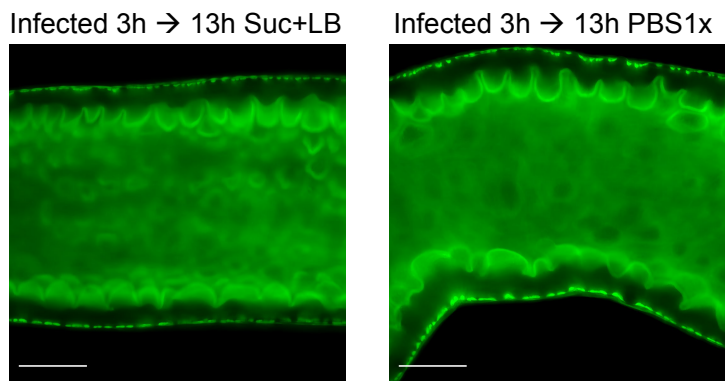

Infected $3 \mathrm{~h} \rightarrow 13 \mathrm{~h} \mathrm{H} 2 \mathrm{O}$

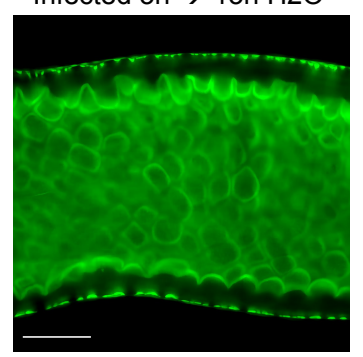

$E$

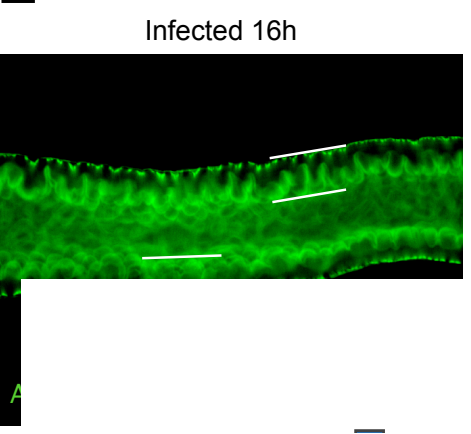

Pre-starved + Infected $16 \mathrm{~h}$

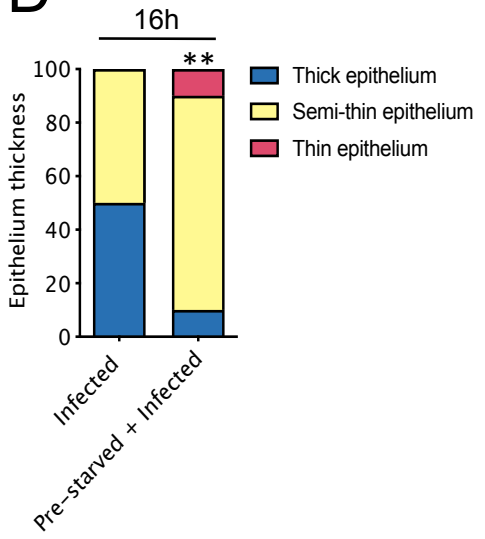

F

G

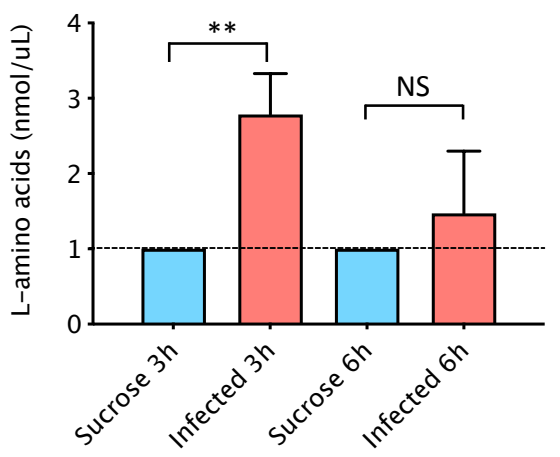




\section{Figure 2}

A

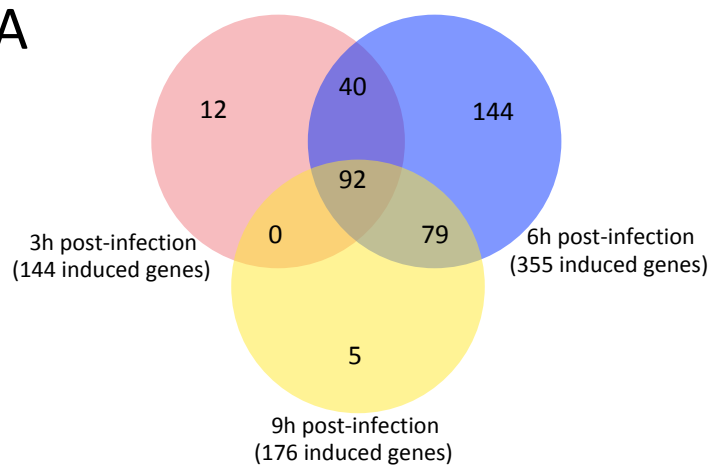

C

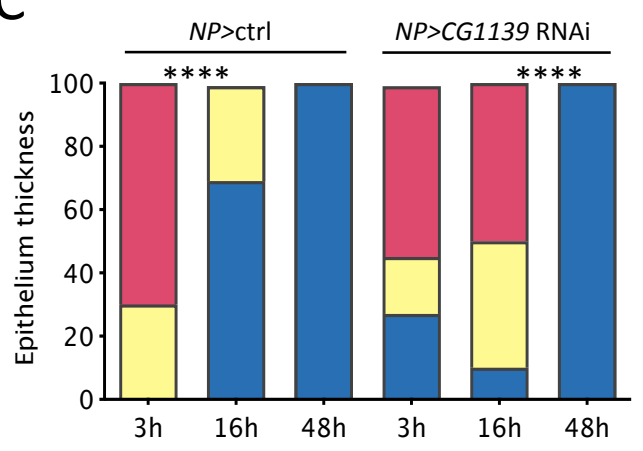

$E$

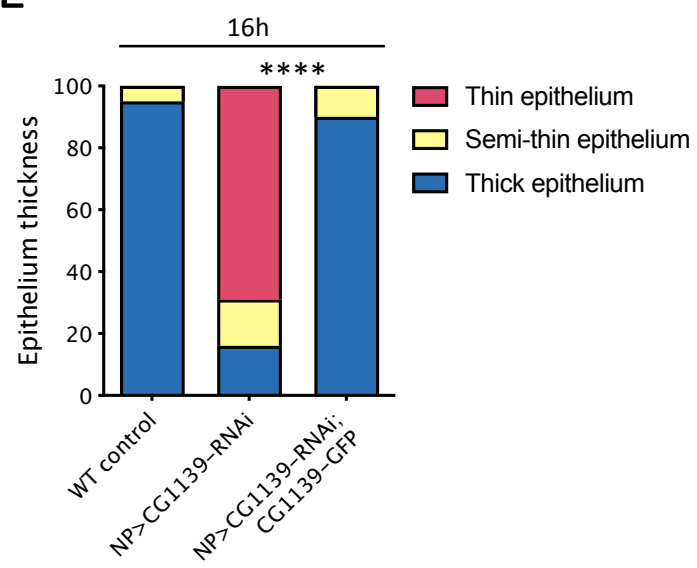

G

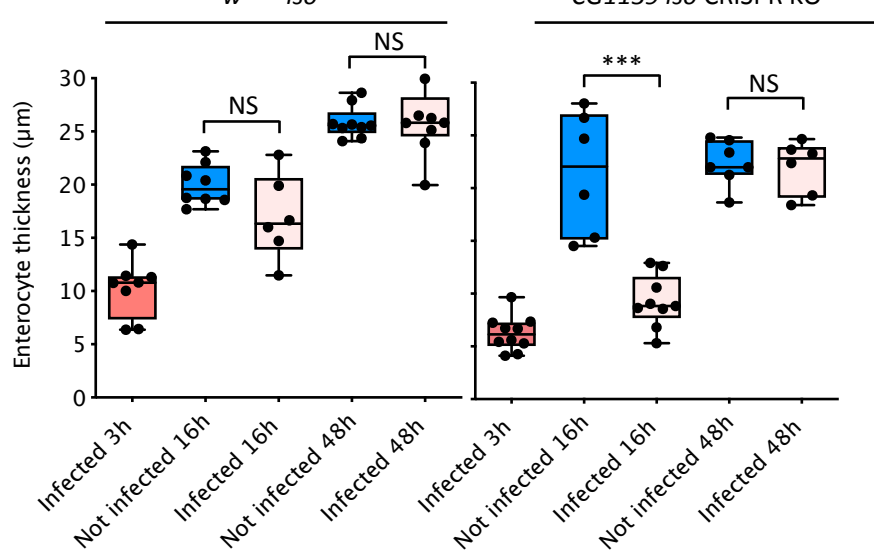

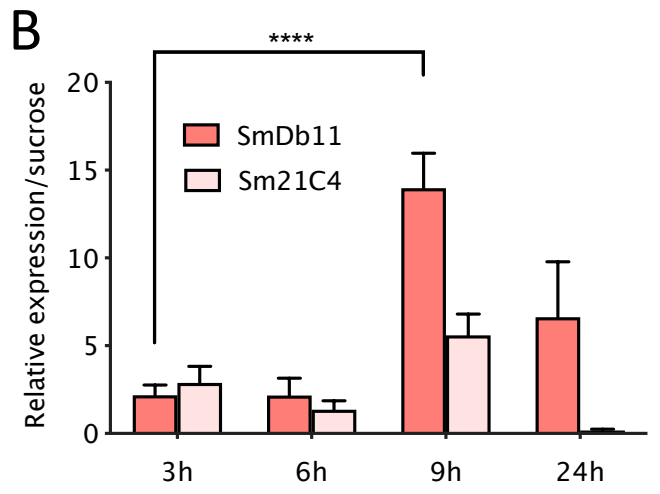

D
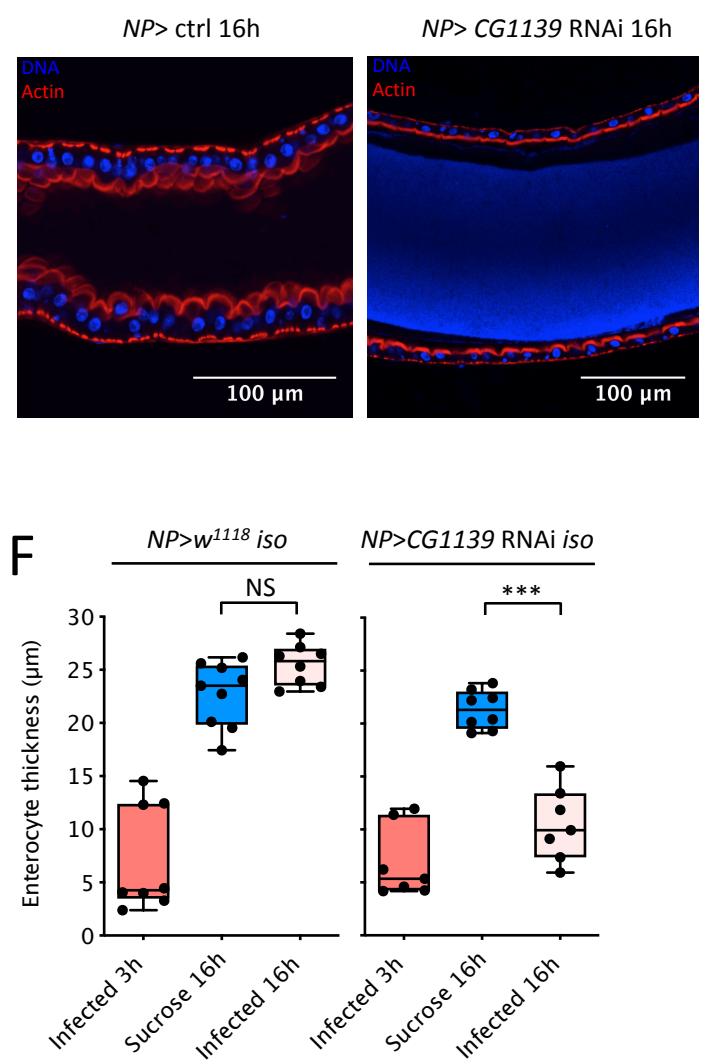

$\mathrm{H}$

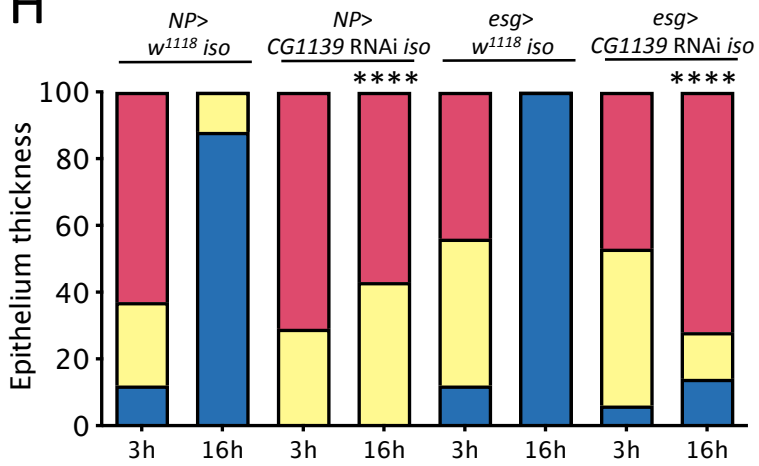


Figure 3

A

Non-infected

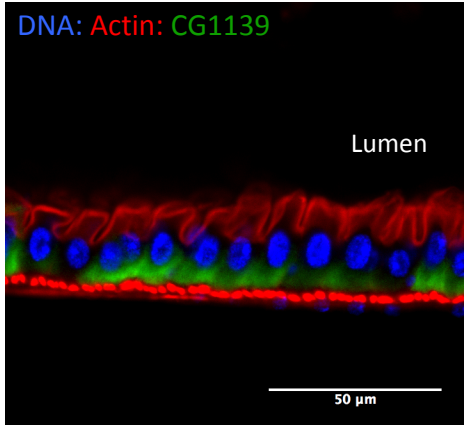

B

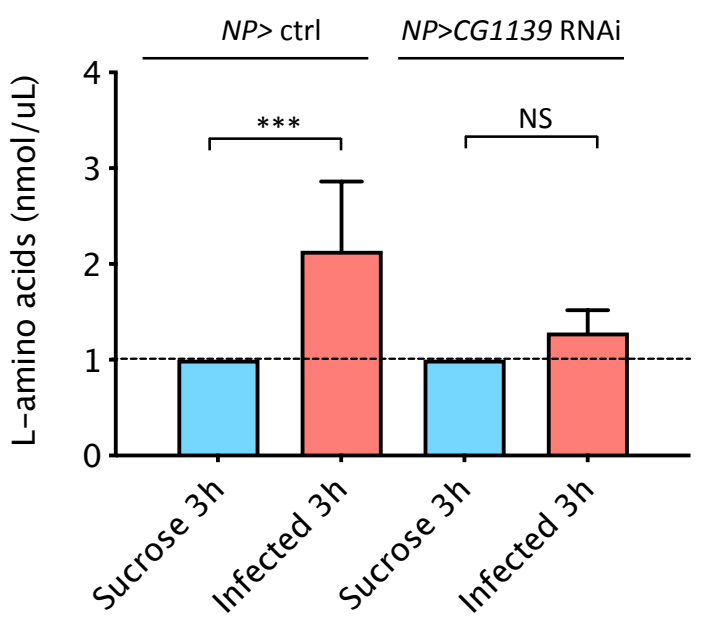

Infected 3h

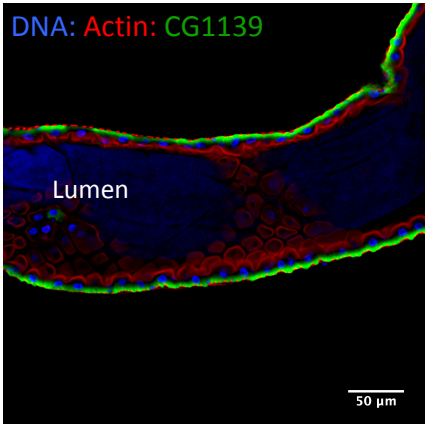

Infected 6h

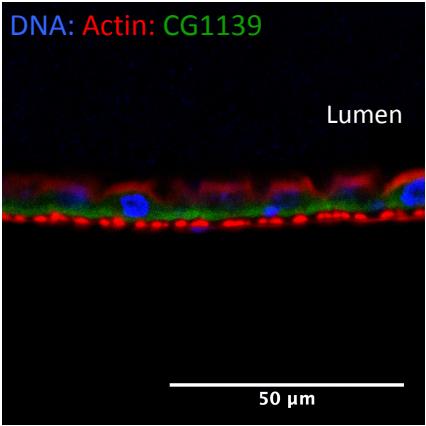

C

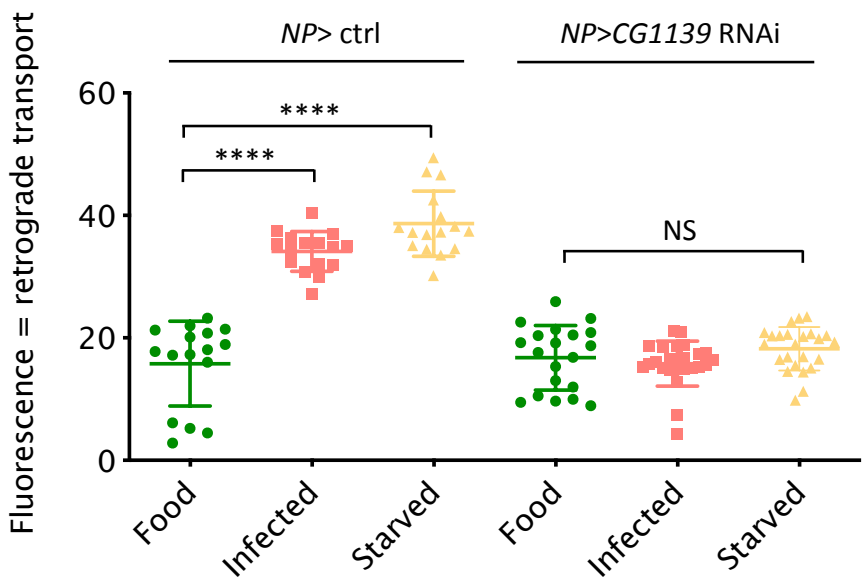




\section{Figure 4}

A

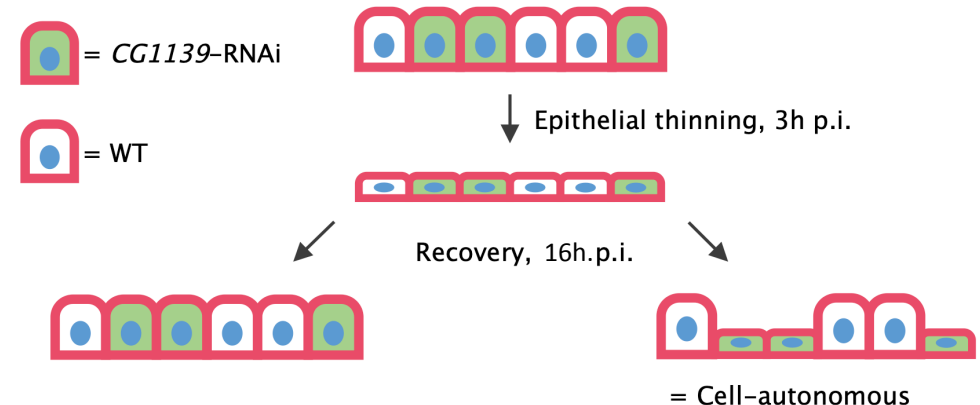

-

$=$ Cell-autonomous

= NON cell-autonomous

B

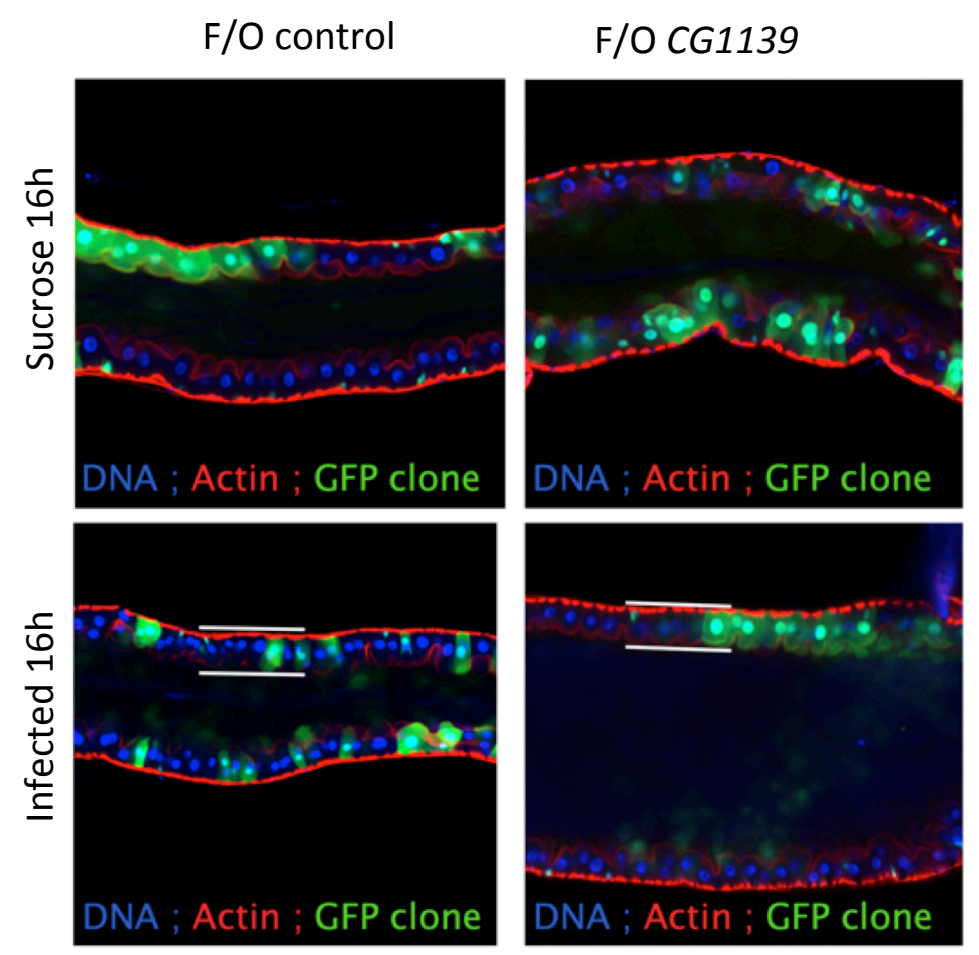




\section{Figure 5}

A

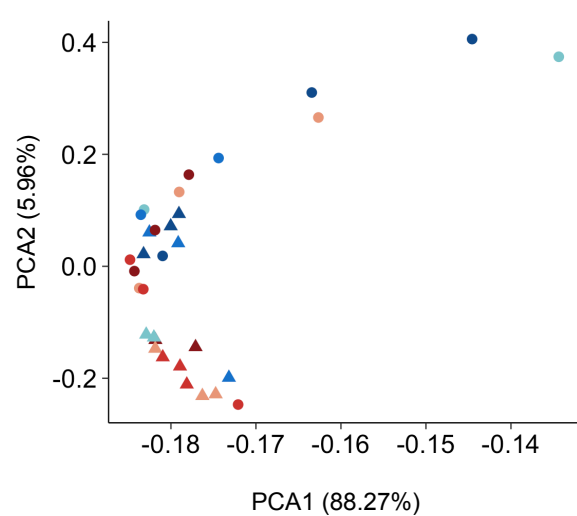

B

Line:

- $N P>\operatorname{ctrl}$

- NP>CG1139 RNAi

Condition:

- Sucrose 3h

- Sucrose $8 \mathrm{~h}$

- Sucrose $16 \mathrm{~h}$

- Infected $3 \mathrm{~h}$

- Infected $8 \mathrm{~h}$

- Infected $16 \mathrm{~h}$

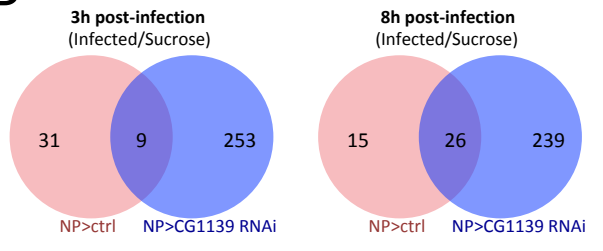

16h post-infection (Infected/Sucrose)

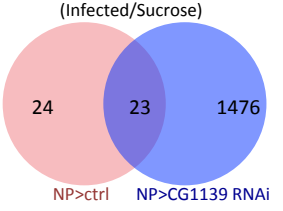

\section{C}

Genes involved in growth differentially regulated in NP> CG1139 RNAi at 16h

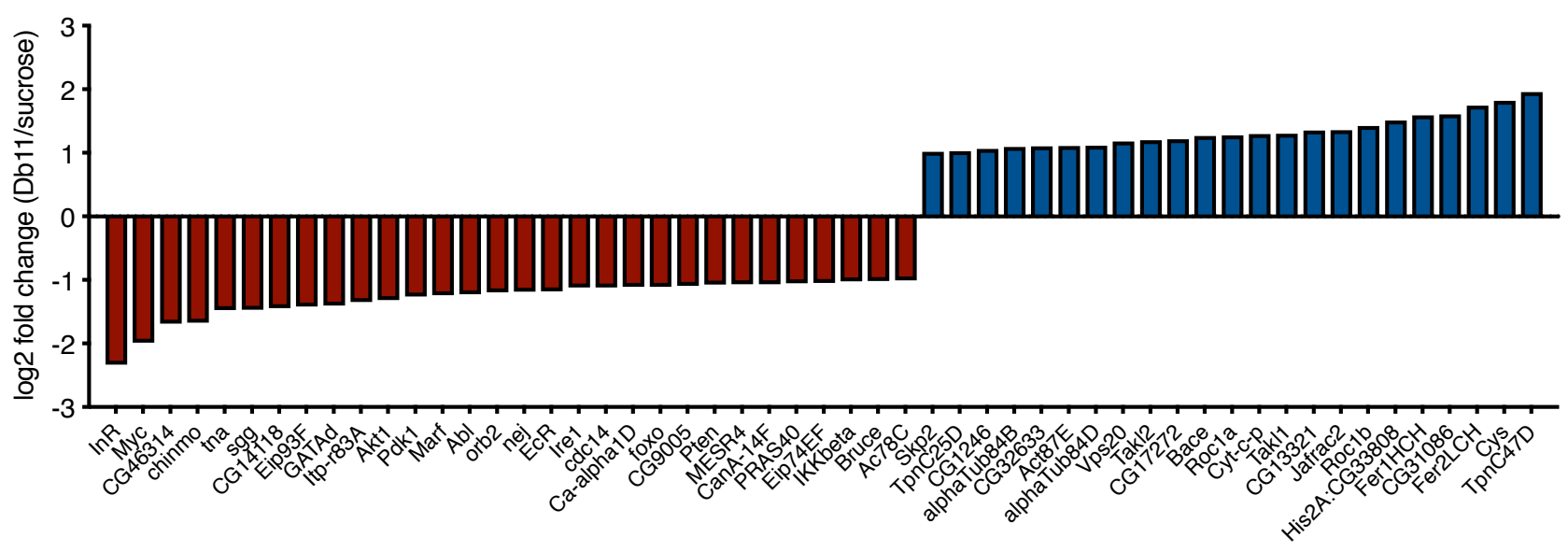

D

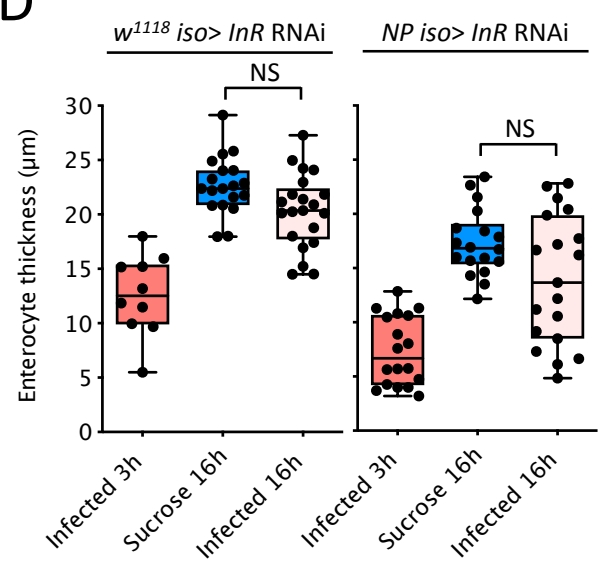

$E$

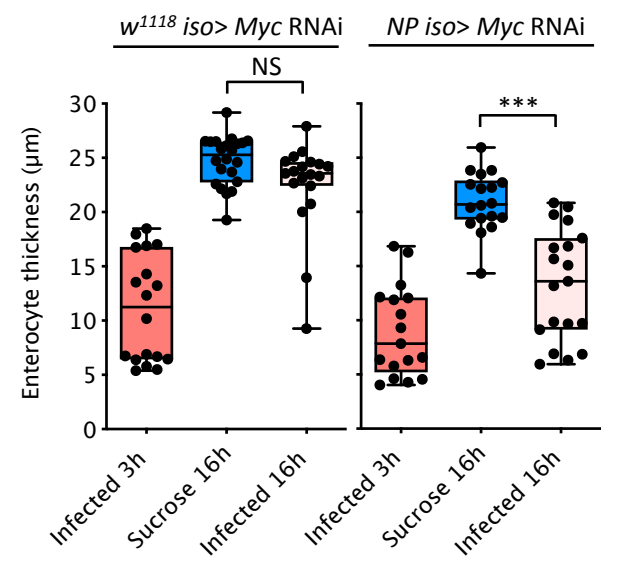

